liche Fürsorge von dem Ideal der individuell betreuenden Hilfe der RGr. abermals weit entfernt und wirkte vor allem als behördlich-schematische, richtsatzzentrier-

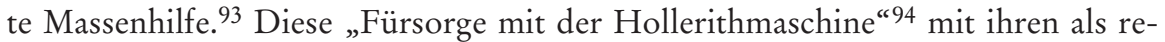
pressiv empfundenen Begleiterscheinungen der rigiden Einkommensüberprüfung und Rückerstattungspflicht war kaum dazu angetan, das ohnehin angeschlagene Image der öffentlichen Fürsorge trotz ihrer unbestreitbaren Erfolge zu verbessern - eine Entwicklung, die seit Ende der vierziger Jahre dieses System zunehmend in die sozialpolitische Defensive drängte.

\title{
2. Fürsorgepolitik im Zeichen des Wiederaufbaus
}

Die Fürsorgepolitik der ersten Nachkriegsjahre in den Westzonen ist gekennzeichnet durch das Bemühen ihrer führenden deutschen Vertreter, das hergebrachte Weimarer System im wesentlichen zu erhalten bzw. wieder zu errichten. Begünstigt wurden diese Bestrebungen schon allein durch die Macht des Faktischen: Mit dem „Dritten Reich“ war auch der bedrohliche Konkurrent NSV untergegangen, während mit den Stadt- und Landkreisen die traditionellen Fürsorgeträger und zumindest die konfessionellen Spitzenverbände bestehen geblieben waren und ihre Zuständigkeit von den Alliierten nicht grundsätzlich in Frage gestellt wurde.

Doch die neuen und sehr ungleich verteilten Lasten der Fürsorge, damit verbunden deren ungleiche Leistungen und neue rechtliche Sonderregelungen, nicht zuletzt auch alliierte Reformbestrebungen drohten das auf RFV und RGr. aufgebaute Fürsorgesystem aus den Angeln zu heben. Die Bemühungen um dessen Konsolidierung vollzogen sich daher auf drei Ebenen: In organisatorischer Hinsicht galten sie dem interessenpolitischen „Überbau“ der Fürsorgeträger in Form der kommunalen Spitzenverbände und des Deutschen Vereins für öffentliche und private Fürsorge (DV) wie einer wirksamen Vertretung gegenüber den Besatzungsmächten, den neuen Länder- und Zoneninstanzen bzw. dem Bund. Auf der finanzpolitischen Ebene standen die Bemühungen um eine gleichmäßigere und zugleich praktikable Verteilung der Lasten zwischen den Fürsorgeverbänden ebenso wie gegenüber den Ländern und Zonen bzw. dem Bund im Vordergrund. Fürsorgerechtlich schließlich zielten diese Bestrebungen auf eine Abwehr alliierter Reformpläne und zunehmender Rechtszersplitterung durch die weitgehende Beibehaltung der Weimarer Rechtsgrundlagen.

93 Vgl. etwa durchaus selbstkritisch die Kommunalvertreter Rudolf Prestel und Heinrich Treibert auf dem Deutschen Fürsorgetag 1949, in: Fürsorge im Dienst, S. 35f., 48ff.; ferner Peter Paul Nahm vom hessischen Innenministerium auf dem Fürsorgetag 1950, in: NDV 30 (1950), S. $241 \mathrm{ff}$.

94 So, zustimmend Hans Achinger zitierend, rückblickend der leitende BMI-Beamte Wilhelm Kitz, in: NDV 31 (1951), S. 133. 


\section{Instanzen und Organisationen der Fürsorgepolitik}

Anders als etwa für das Gebiet des Arbeitswesens bildeten die westlichen Alliierten für den Bereich der Wohlfahrtspflege im Laufe der Nachkriegszeit keine eigenständigen zonalen oder überzonalen Verwaltungs- und Legislativinstanzen, sondern beließen die Zuständigkeit bei den Militärregierungen bzw. den Ländern. In der US-Zone erhielten die Länder bereits bei ihrer Gründung im Herbst 1945 eigene, wenn auch von der Militärregierung kontrollierte, legislative und exekutive Befugnisse; während in Bayern und Württemberg-Baden das Fürsorgewesen bei den Innenministerien ressortierte, unterstand es in Hessen dem Minister für Arbeit und Wohlfahrt. ${ }^{95}$ Mit dem „Ausschuß für Wohlfahrtswesen“ wurde auf Wunsch der amerikanischen Militärregierung aus Fachbeamten der Länder und Kommunen und Vertretern der freien Wohlfahrtsverbände im Mai 1946 beim Stuttgarter Länderrat ein zonales Gremium geschaffen, das außerdem die Zusammenarbeit mit den betroffenen Stellen der britischen Zone suchen sollte. ${ }^{96}$

Auch in der britischen Zone wurde Anfang 1946 aus Vertretern der Provinzen und Länder, der Fürsorgeverbände, der Spitzenverbände der freien Wohlfahrtspflege und des Roten Kreuzes beim Zonenbeirat ein Wohlfahrtsausschuß gebildet, der unter britischem Vorsitz die Militärregierung in Fürsorgefragen beriet. ${ }^{97}$ Nachdem Ende 1946 die Zuständigkeit für Wohlfahrtsaufgaben auf die neu gegründeten Länder übergegangen war ${ }^{98}$, diente der Ausschuß - nun unter deutschem Vorsitz - der Beratung der zuständigen deutschen Stellen und entwickelte sich allmählich zu einem informellen Koordinierungsorgan der Länder zur Erarbeitung zoneneinheitlicher Reformvorschläge, seit 1948 dann auch in Koordination mit dem Sozialpolitischen Ausschuß des Zonenbeirats. Wie der Wohlfahrtsausschuß der amerikanischen Zone war auch derjenige der britischen Zone bis zur Bildung der Bundesregierung tätig. ${ }^{99}$ Als weiteres länderübergreifendes Gremium fungierte außerdem die Arbeitsgemeinschaft der Fürsorgedezernenten der britischen Zone, doch das Fehlen länderübergreifender Verwaltungsinstitutionen konnten diese Ausschüsse nicht kompensieren, so daß es auf dem Gebiet des Fürsorgerechts zu erheblichen Sonderentwicklungen kam.

Auf seiten der Kommunen als den Trägern der Fürsorge knüpfte die organisatorische Formierung auch jenseits der eigentlichen Aufgabendurchführung durch die Fürsorgeverbände an Weimarer Traditionen an, indem mit den (natürlich nicht primär fürsorgepolitisch orientierten) kommunalen Spitzenverbänden wie mit dem DV traditionelle Akteure der Fürsorgepolitik erneut die Bühne betraten. Nach Verbot und Auflösung der nationalsozialistisch dominierten Einheitsorga-

95 Vgl., auch für das Folgende, Preller, Sozialpolitik, S. 76ff.; Heisig, Armenpolitik, 1995, S. 28f.; Walter Vogel, Westdeutschland, S. 575f.; ferner Härtel, Länderrat, S. 15, $158 f$.

96 Vgl. Tagung des Länderrates am 5.3.1946, in: Akten zur Vorgeschichte, Bd.1, S. 325; Schreiben des Generalsekretärs des Länderrats Rossmann an den württemberg-badischen Bevollmächtigten Gögler, 10.5.1946, Abschrift, ADW, ZB 295.

97 Vgl. auch Dorendorf, Zonenbeirat, S. 28, 118.

98 Vgl. Verordnung Nr. 57 vom 1.12.1946, Amtsblatt der Militärregierung Deutschland. Britisches Kontrollgebiet. No.15, S.344-346.

99 Vgl. NDV 28 (1948), S. 174; Härtel, Länderrat, S. 80, 83; Walter Vogel, Westdeutschland, S. 576 . 
nisation des Deutschen Gemeindetages kamen weitere Pläne für eine neue gemeinsame Spitzenorganisation der Kommunen nicht mehr zum Tragen; vielmehr betrieben zahlreiche Kommunalpolitiker und ehemalige Mitarbeiter der Kommunalabteilung des alten Reichsinnenministeriums von der britischen Zone aus die Neugründung der wichtigsten ehemaligen separaten Spitzenverbände.

Als erster Verband entstand auf Initiative des damaligen Kölner Oberbürgermeisters Konrad Adenauer der Deutsche Städtetag wieder ${ }^{100}$; unter Leitung seines Nachfolgers in Köln, Hermann Pünder, konstituierte sich der Städtetag im Dezember 1945 zunächst in der britischen Zone, seit Anfang 1947 wieder als „Deutscher Städtetag“ (DST), der auch die Städte der US-Zone repräsentierte und damit eine der ersten zonenübergreifenden Organisationen nach 1945 überhaupt bildete. ${ }^{101}$ Die Landesverbände der französischen Zone konnten sich offiziell erst im Frühling 1949 anschließen. Anders als andere kommunale Spitzenverbände war der DST keine reine Dachorganisation seiner Landesverbände, sondern organisierte neben den kreisfreien und einer Minderheit kreisangehöriger Städte auch die drei Stadtstaaten und damit Bundesratsmitglieder als direkte Mitglieder; das verkürzte die Entscheidungswege und erhöhte die Effektivität und politische Durchschlagkraft der bald gut ausgebauten Kölner Hauptgeschäftsstelle. Der Verband verstand sich - wie auch die anderen kommunalen Spitzenverbände - als überparteilicher Repräsentant allgemeiner kommunaler Bürgerinteressen gegenüber Ländern und Zentralstaat und erstrebte eine Stärkung der kommunalen Selbstverwaltungskompetenzen; er entwickelte sich bald wieder zu einem einflußreichen Interessenorgan städtischer kommunaler Belange, auch und gerade in der Fürsorgepolitik. Freilich war und ist der DST genauso wenig wie die anderen Spitzenverbände ein in sich homogener Interessenblock: Zum einen sind die verschiedenen und nicht selten gegenläufigen Interessen der einzelnen kommunalen Politikbereiche zu koordinieren, die sich auch in der Ressort- und Gremienaufteilung der Geschäftsstellen widerspiegeln. So verfolgte etwa der Finanzausschuß des DST bei der Fürsorgereform durchaus eine andere Linie als der federführende Sozialausschuß und der Beigeordnete für Soziales. Außerdem vereinigt der DST unter seinem Dach nicht nur Mitglieder höchst unterschiedlicher Struktur - von den Großstädten einschließlich der Stadtstaaten bis hin zu kleinen kreisfreien Städten wie Schnackenburg mit einigen hundert Einwohnern -, sondern auch

100 Geschichte und Einfluß der kommunalen Spitzenverbände in den ersten Jahren der Bundesrepublik sind bislang noch nicht umfassend auf breiter empirischer Grundlage aufgearbeitet; abgesehen von einigen älteren politikwissenschaftlichen Arbeiten - vgl. etwa Bertram, Staatspolitik; Voigt, Partizipation - und Untersuchungen zu heutigen Einflußmöglichkeiten - vgl. Jaedicke u.a., Politik - dominieren hier offiziöse Verbandsgeschichten: Zur Wiedergründung und Geschichte des Deutschen Städtetages nach 1945 vgl. ausführlich Ziebill, Geschichte, S. 66ff.; ferner Weinberger, Städtetag, S.13ff., sowie jüngst Meyer/Meyer-Woeller, 100 Jahre, S. 57ff. Zur Geschichte des Deutschen Landkreistages nach 1945 siehe (in den Datierungen leider oft unzuverlässig) Groeben/Heide, Geschichte, S.215ff.; zur Frühgeschichte der kommunalen Spitzenverbände sowie zu den persönlichen Verbindungen wichtiger kommunaler Spitzenfunktionäre seit der Zeit des Nationalsozialismus siehe Heisig, Armenpolitik, 1995, S.28, sowie den biographischen Anhang, in: ders., Armenpolitik, 1990, S. $523 \mathrm{ff}$.

101 Vgl. Deutschland-Jahrbuch 1949, S. 47. 
unterschiedlicher parteipolitischer Orientierung, was ebenfalls auf die Meinungsbildung in den Verbandsgremien durchschlägt. In den fünfziger und sechziger Jahren wurde der DST parteipolitisch von der SPD mit ihrer traditionellen Stärke in den deutschen Großstädten dominiert, wenn auch eine Satzungsbestimmung, wonach in allen Gremien alle Beschlüsse mit einer Dreiviertel-Mehrheit zu fassen waren, für einen gewissen Ausgleich sorgen konnte. ${ }^{102}$

Als zweiter kommunaler Spitzenverband nahm ebenfalls zuerst in der britischen Zone im Juli 1946 der „Deutsche Städtebund“ (DSB) in Nachfolge des ehemaligen Reichsstädtebundes seine Arbeit auf. Er organisierte ausschließlich kreisangehörige Städte und größere, stadtähnliche Gemeinden mit Ausnahme von Bayern und Baden. ${ }^{103}$ Trotz der engen Zusammenarbeit mit Vertretern des DST bei Gründung und Aufbau des Verbandes konnten sich die beiden Spitzenvertretungen der Städte wie in den zwanziger Jahren auch nach 1945 nicht auf einen $\mathrm{Zu}$ sammenschluß einigen. ${ }^{104}$

Die Wiedergründung des „Deutschen Landkreistages“ (DLT) ging zunächst von Hessen aus, wo der Ziegenhainer Landrat und spätere erste Präsident des DLT, Heinrich Treibert, seit Sommer 1945 die Zusammenarbeit der Landkreise forcierte. ${ }^{105}$ Parallel dazu betrieben ehemalige Mitarbeiter des DLT bzw. des aufgelösten Deutschen Gemeindetages die Gründung eines Landkreistages in der britischen Zone im September 1946; im Februar 1947 konstituierte sich der DLT für die gesamte Bizone. Der Verband ist anders als sein städtisches Pendant streng föderal strukturiert, indem er als Dachverband der einzelnen, auf ihr Eigenleben bedachten Landesverbände fungiert und die Landkreise nur indirekt organisiert. Parteipolitisch war der DLT nach Ablösung des Sozialdemokraten Treibert 1949 fest in den Händen der CDU/CSU. Da die Vorsitzenden der acht Landesverbände automatisch das Präsidium bildeten und die SPD in ländlichen Gebieten traditionell schwach repräsentiert war, gelang es ihr viele Jahre nicht, dort überhaupt einen Sitz zu erlangen. Präsident und Hauptgeschäftsführer wurden ohnehin von der Union gestellt. ${ }^{106}$

Mit dem „Deutschen Gemeindeverband“ formierte sich unter hessischer Federführung im November 1947 als letzter kommunaler Spitzenverband auch die Organisation der kreisangehörigen Gemeinden zunächst in der Bizone in lockerer Form neu; auch ihm konnten sich die mittlerweile in der französischen Zone gebildeten Landesverbände erst im Laufe des Jahres 1949 anschließen. ${ }^{107}$ Seit 1950

102 Der starke Einfluß der SPD hing auch damit zusammen, daß nach dem Kölner Oberbürgermeister Hermann Pünder (CDU) bis 1963 üblicherweise Berlins Regierende Bürgermeister und damit führende SPD-Politiker das Amt des DST-Präsidenten innehatten. Mit Otto Ziebill war ab 1951 ebenfalls ein Mann der SPD Hauptgeschäftsführer des DST, und im DST-Präsidium hatte die SPD bis Mitte der sechziger Jahre ebenfalls ein deutliches Übergewicht. Zu Struktur, Organisation und politischer Ausrichtung der kommunalen Spitzenverbände vgl. insgesamt Jaedicke u.a., Politik, S.29ff.; Bertram, Staatspolitik, S. 82ff.

103 Vgl. Mombauer, Städte- und Gemeindebund, S. 494.

104 Vielmehr fusionierte der Deutsche Städtebund 1973 mit dem Deutschen Gemeindetag.

105 Vgl. auch zum Folgenden Groeben/Heide, Geschichte, S. 226ff.

106 Vgl. Jaedicke u.a., Politik, S. 39.

107 Vgl. Göb, 50 Jahre, S. 34ff. 
in „Deutscher Gemeindetag“ (DGT) umbenannt und seit 1951 als fester Verband organisiert, bildete er einen Dachverband der einzelnen Landesverbände der kreisangehörigen Städte, Ämter und Gemeinden. Nach Ausscheiden von Wilhelm Mellies (SPD) 1953 fungierte von 1954 bis 1966 der spätere Bundesminister Paul Lücke (CDU) als Präsident des DGT.

Abgesehen von den unterschiedlichen parteipolitischen Präferenzen besteht zwischen den einzelnen Spitzenverbänden eine teils historisch, teils durch Interessenunterschiede und Prestigeprobleme begründete Konkurrenz, wobei traditionell der DST auch dank strafferer Organisation und finanzieller Möglichkeiten eine beherrschende Stellung innehatte. ${ }^{108}$ Doch im „Interesse einer kraftvollen Vertretung der kommunalen Selbstverwaltung "109 bildeten die vier Spitzenverbände 1947/48 wie in den letzten Jahren der Weimarer Republik zunächst in lockerer Form die „Deutsche Arbeitsgemeinschaft der kommunalen Spitzenverbände“, im Mai 1953 dann die kaum fester organisierte „Bundesvereinigung der Kommunalen Spitzenverbände“. Unter Federführung des DST fungiert diese vor allem als gemeinsames offizielles Sprachrohr der Kommunen im politischen Entscheidungsprozeß, deren Wirkung im Einzelfall allerdings stark von der Bereitschaft der einzelnen Spitzenverbände zu einmütiger Stellungnahme abhängt. ${ }^{110}$ Seit Ende 1947 also verfügten die Kommunen und damit die Träger der öffentlichen Fürsorge zumindest in der Bizone wieder über jeweils eigene Organisationen zur Bündelung und Geltendmachung ihrer Belange gegenüber Länderund Zonen- bzw. Bundesinstanzen, wobei sie jedoch damals wie heute Wert darauf gelegt haben, nicht als Interessenverband im herkömmlichen Sinne zu gelten, sondern als „öffentliche Einrichtungen“ zur Vertretung öffentlicher Interessen. ${ }^{111}$

Auch die Landesfürsorgeverbände der britischen und amerikanischen Zone bildeten Ende August 1947 eine eigene Arbeitsgemeinschaft, die, später auf die französische Zone ausgedehnt, oft in enger Kooperation mit DST, DLT und DV sich vor allem in Fragen der fürsorgerischen Lastenverteilung und Organisationsstruktur Gehör zu verschaffen wußte. Schließlich gründeten auch die freien Wohlfahrtsverbände mit der „Arbeitsgemeinschaft der Spitzenverbände der freien Wohlfahrtspflege“ im Oktober 1948 einen Zusammenschluß, dem sich anders als bei seinem Weimarer Vorläufer nun auch die AWO anschloß.112 Die von den konfessionellen Verbänden bereits seit 1947 betriebene Kooperation verdankte sich in erster Linie den wirtschaftlichen Schwierigkeiten, denen sich die Wohlfahrtsverbände vor allem seit der Währungsreform gegenübersahen. Hauptaufgabe der Arbeitsgemeinschaft blieb daher für lange Zeit die Erschließung neuer Finanzmittel. Darüber hinaus wurde sie bereits von der ersten Bundesregierung 1949 anerkannt und zur Gutachterin bei der Beratung von Wohlfahrtsgesetzen bestellt.

108 Vgl. Jaedicke u.a., Politik, S. 39f.; Voigt, Partizipation, S. 21.

109 Ziebill, Geschichte, S.282; zum Folgenden vgl. ebenda, S. 282f.; Groeben/Heide, Geschichte, S. 238f.

110 Vgl. Bertram, Staatspolitik, S. 90ff.; Voigt, Partizipation, S. $20 \mathrm{f}$.

111 So Groeben/Heide, Geschichte, S. 235; ähnlich Ziebill, Geschichte, S. $322 \mathrm{ff}$.

112 Vgl. Hammerschmidt, Wohlfahrtsverbände in der Nachkriegszeit, S. $76 f f$. 
Ein ganz entscheidender Schritt der organisatorischen Neuformierung des fürsorgepolitischen Systems war die Wiedererrichtung des DV seit $1946 .{ }^{113}$ Treibende Kraft war hier der ehemalige und bald erneut bestätigte DV-Vorsitzende Wilhelm Polligkeit ${ }^{114}$, seit den frühen Tagen der Weimarer Republik eine der zentralen Figuren der deutschen Fürsorgepolitik. Zusammen mit der ehemaligen DVGeschäftsführerin und seiner späteren Frau Hilde Eiserhardt, unterstützt vom Frankfurter Oberbürgermeister Kurt Blaum, organisierte er schon seit Sommer 1945 in Hessen in Fühlungnahme mit dortigen Kommunalvertretern und ausgewiesenen Fürsorgeexperten wie Hans Achinger den Wiederaufbau des Vereins. ${ }^{115}$ Auch dank Polligkeits guten persönlichen Kontakten zu den amerikanischen Militärbehörden wurde der DV am 11. April 1946 für Großhessen wieder zugelassen, so daß im Mai ein „Fürsorgetag“ in Nachfolge der traditionellen DV-Fürsorgekongresse abgehalten werden konnte. Im Laufe des Jahres folgte, von den zuständigen US-Behörden gefördert, die Zulassung auch für Bayern und Württemberg-Baden, 1947 dann für die Länder der britischen, 1948 für die französische Zone, 1949 schließlich für West-Berlin.

Finanziert wurde der Neuaufbau des DV u.a. durch Mittel der Landesregierungen sowie einzelner Kommunen und Vereinigungen der freien Wohlfahrtspflege; Ende der fünfziger Jahre wurde die Arbeit des DV dann zu etwa zwei Dritteln von Bund, Ländern, Städten und Landkreisen getragen. ${ }^{116}$ Nach improvisierten Anfängen entwickelte sich der DV bald wieder zu einer einflußreichen Organisation mit Geschäftsstelle in Frankfurt, hauptamtlichem Vorsitzenden, Geschäftsführer und Fachreferenten, eigener Bibliothek, Fortbildungseinrichtungen und umfangreicher Publikationstätigkeit, vor allem mit dem Verbandsorgan des „Nachrichtendienstes“ (NDV). Bereits 1949 hatte der DV wieder mehr als 1300 Mitglieder. ${ }^{117}$

113 Vgl. Willing, Vorgeschichte, S. 603ff. Für einen Überblick über die Geschichte des Deutschen Vereins allgemein die akklamatorische Festschrift zu dessen 100jährigem Jubiläum von Eberhard Orthbandt, Deutscher Verein; in kritischer Auseinandersetzung damit: Tennstedt, Fürsorgegeschichte; ferner zu einzelnen Epochen: Krug von Nidda, Polligkeit; Sachße/Tennstedt, Geschichte, Bd.2, passim, sowie Bd.3, S.147f.; Hansen, Wohlfahrtspolitik, S. $87 \mathrm{ff}$.

114 Der Jurist Wilhelm Polligkeit (1876-1960) war Geschäftsführer der Centrale für private Fürsorge von Wilhelm Merton in Frankfurt a.M. gewesen, ehe er 1920 die hauptamtliche Geschäftsführung des DV übernahm und 1922 dessen, den Verein von nun an prägender Vorsitzender wurde. Polligkeit, der sich nach 1933 den neuen Verhältnissen anzupassen suchte, allerdings nicht in die NSDAP eintrat, wurde 1935 als Vorsitzender abgesetzt, fungierte noch bis 1936 als Geschäftsführer und betätigte sich anschließend bis Kriegsende wissenschaftlich im Rahmen einer Honorarprofessur an der Frankfurter Universität. Im September 1945 wurde er hauptamtlicher Stadtrat und Fürsorgedezernent in Frankfurt. Vorsitzender des DV war er erneut von 1946 bis 1950; zur Vita Polligkeits vgl. Dörrie, Polligkeit; Tennstedt, Fürsorgegeschichte, S. 80ff., $96 \mathrm{ff}$.

115 Vgl., auch für das Folgende, Krug von Nidda, Polligkeit, S. 249ff.; ferner den Rückblick von Heinrich Treibert in: NDV 31 (1951), S.128f.; Rundschreiben 1946, S.1; Rundschreiben 1947, S.1; Begrüßungsansprache Polligkeits in: Aufgaben der Fürsorge, S. 9; NDV 28 (1948), S.1f.; 30 (1950), S.239; Polligkeit-Eiserhardt/Pense, Ziele, S.468ff.; Schrapper, Hans Muthesius, S. 182ff.; Heisig, Armenpolitik, 1995, S. $29 f$.

116 Vgl. Pense, Bericht, S. 448.

117 Vgl. Eröffnungsansprache Polligkeits in: Fürsorge im Dienst, S. 5. 
1880 gegründet verstand und versteht sich der DV als politisch neutraler Fachverband, der laut Satzung von 1951 einen „Mittelpunkt für alle in Deutschland auf dem Gebiet der öffentlichen und freien Wohlfahrtspflege und der Sozialreform hervortretenden Bestrebungen“ bilden sollte. ${ }^{118}$ Hauptaufgaben des DV waren danach die Beeinflussung von fürsorgerischen Reformvorhaben, wissenschaftliche Information und praktische Beratung seiner Mitglieder, die Erstellung von Fachgutachten sowie die „Verbreitung gesunder Grundsätze in der Praxis der öffentlichen und freien Wohlfahrtspflege“. ${ }^{119}$ Wie in der Weimarer Republik erweist sich der DV auch nach 1945 als ein von herkömmlichen Verbandsdefinitionen nicht ohne weiteres zu erfassendes Konstrukt, da er unter dem Dach eines privatrechtlich organisierten Vereins bald wieder die große Mehrheit der mit der Wohlfahrtspflege befaßten Institutionen, Körperschaften und Personen vereinigte: Bundesministerien, Landes- und Kommunalbehörden ebenso wie die Spitzenverbände der Kommunen und der freien Wohlfahrtspflege, karitative Spezialorganisationen und Berufsverbände ebenso wie Abgeordnete, Wissenschaftler und Sozialdezernenten, darüber hinaus Vertreter anderer Sozialleistungsträger, von Gewerkschaften und Arbeitgeberverbänden, gemeinnützigen Betrieben und Wohlfahrtsschulen. Diese für den Fürsorgebereich in Deutschland typische personale und organisatorische Verflechtung von legislativen, exekutiven und sozialwissenschaftlichen Instanzen erlaubt es somit nicht, den DV im fürsorgepolitischen Kräftemessen als Lobby für klar definierte Interessenlagen zu verstehen, zumal er in seinem Leitungsorgan führende Sozial- und Kommunalpolitiker der verschiedenen Parteien vereinte. ${ }^{120}$ Inwieweit der DV in seiner Rolle „einer grauen Eminenz der Fürsorgepolitik“121 die selbst gestellte Aufgabe des unpolitischen Fachberaters der Politik erfüllen konnte, erscheint allerdings fraglich, beriet der DV doch diejenigen, die in seine eigenen Reihen gehörten und dort die fürsorgepolitische Ausrichtung des Vereins mitbestimmten. In der Forschung ist der DV daher als „,Koordinierungskartell' der gesamten öffentlichen und privaten Wohlfahrtspflege"122 bezeichnet, seine Fachlichkeit gerade im Zusammenhang der Warenkorb-Regelsatzproblematik seit Ende der siebziger Jahre stark angezweifelt ${ }^{123}$ und ferner auf die zwangsläufige Kompromißhaftigkeit seiner zentralen Äußerungen verwiesen worden. ${ }^{124}$ Letzteres verbietet es allerdings auch, ihn als reinen Sachwalter der Kommunen zu sehen, wenn auch für die Gemeinden unannehmbare Beschlüsse in der Regel nicht zustande kommen und diese den Verband als

118 Vgl. $\ 2$ der Satzung des Deutschen Vereins für öffentliche und private Fürsorge, wie auf dem Fürsorgetag 1951 beschlossen, in: Beiträge zur Entwicklung, S. 490.

119 Ebenda.

120 Vgl. etwa die Liste der Mitglieder des DV-Vorstands vom Oktober 1950, NDV 30 (1950), S. 240, dem neben der CDU-Bundestagsabgeordneten Helene Weber auch die Berliner Bürgermeisterin Louise Schroeder, der niedersächsische Arbeitsminister Heinrich Albertz und DGB-Vorstandsmitglied Willi Richter (alle SPD) angehörten.

121 Jaedicke u.a., Politik, S. 44.

122 Hofmann/Leibfried, Regelmäßigkeiten, S. 262.

123 Vgl. Rudloff, Fürsorge, S. 198f., mit weiteren Verweisen; Jaedicke u.a., Politik, S. 81ff.; ferner die im Literaturverzeichnis angeführten Arbeiten von Stephan Leibfried, Eckhard Hansen, Albert Hofmann und Michael Heisig.

124 So bereits Schäfer, Rolle, S. 260, Anm. 892; ferner Winter, Interessen, S. $284 \mathrm{ff.}$ 
neutrales Werbeinstrument für eigene Vorschläge nutzen können. ${ }^{125}$ In den späten vierziger und den fünfziger Jahren bildete der DV mit seinen Fürsorgetagen und Fachausschüssen jedenfalls noch die unangefochtene Hauptplattform für die Überlegungen zur Reform des Fürsorgerechts.

Auf dem Gebiet des Fürsorgewesens war damit die organisatorische und institutionelle Konsolidierung in enger Anlehnung an Weimarer Vorbilder bis zur Gründung der Bundesrepublik weitgehend erreicht. Diese institutionellen Konstellationen unterscheiden sich teilweise ganz erheblich von denen in anderen Bereichen der sozialen Sicherung und bedingten damit für mehrere Jahrzehnte den Prozeß der politischen Interessenvermittlung und Entscheidungsfindung im Bereich der Fürsorge bzw. Sozialhilfe ${ }^{126}$ : Die Interessen der tatsächlichen oder potentiellen Leistungsempfänger waren kaum zu bündeln und in einer schlagkräftigen Klientelorganisation zu organisieren; das galt erst recht vor 1961, als aufgrund der relativ geringen Verrechtlichung und Standardisierung dieses Systems und der starken Fluktuation der Empfänger sich kaum gleichgerichtete Interessenlagen herausbilden und damit auch bestimmte Wählerschichten angesprochen werden konnten. ${ }^{127} \mathrm{Ob}$ dieser Mangel an Organisierbarkeit durch „mittelbare Interessentransformation“ über eine eigentlich anderen Zielen verpflichtete Organisation, wie sie die Gewerkschaften für die Rentner darstellen ${ }^{128}$, auch bei der Fürsorgereform der fünfziger Jahren aufgefangen wurde, wird im Folgenden zu klären sein.

Eine weitere Besonderheit liegt darin, daß mit den Kommunen und den Landesfürsorgeverbänden öffentlich-rechtliche Institutionen als Träger dieses Sozialsystems fungierten, die in den Staatsaufbau inkorporiert waren und damit über einen besonderen Zugang zu den Entscheidungsebenen in Bund und Ländern verfügten. ${ }^{129}$ Vor allem aber waren sie nicht - wie etwa die Versorgungsbehörden weisungsgebundene Exekutivorgane, sondern verwaltungsmäßig und politisch eigenständige Körperschaften mit eigener demokratischer Legitimation, denen der Bund, aber auch die Länder Freiräume zur eigenständigen Gestaltung des Fürsorgewesens lassen mußten, sollte dieser Bereich ihrem verfassungsmäßig verbürgten

125 Vgl. Jaedicke u.a., Politik, S. 45f.

126 Mit der Auswirkung sozialrechtlicher Besonderheiten einzelner Sicherungssysteme auf die Struktur von Politiknetzwerken befaßt sich Winter, Interessen, und bearbeitet dabei auch den Bereich der Sozialhilfe, allerdings erst lange nach Verabschiedung des BSHG. $\mathrm{Da}$ aber wichtige Elemente gerade des institutionellen Gefüges das Fürsorgesystem auch vor 1961 schon kennzeichneten, lassen sich seine Überlegungen teilweise auch für die fünfziger Jahre nutzen, ohne den Anspruch zu erheben, ein politikwissenschaftliches Erklärungsmodell auf diese Weise historisch verifizieren zu können, zumal Winter die kommunalen Spitzenverbände wie die Ebene der Parteien aus seinen Überlegungen de facto ausklammert und generell von einer "Ausgliederung sozialpolitischer Steuerungsaufgaben aus dem Staatsapparat im engeren Sinne“ hinein in "Implementationsagenturen“ spricht, zu denen er auch die kommunalen Sozialhilfeträger zählt (S.358). Historisch kann für die Fürsorge aber genau umgekehrt nur von einer zunehmenden Eingliederung in die staatliche Steuerung gesprochen werden.

127 Vgl. ebenda, S. 89ff., 102ff., 425ff.

128 Vgl. ebenda, S. $138 \mathrm{ff}$., $443 \mathrm{ff}$.

129 Vgl. ebenda, S. 418. 
Selbstverwaltungsrecht zugeordnet bleiben. Im Gegensatz etwa zu den Sozialversicherungsträgern konzentrierte sich das politische Interesse der Kommunen dabei keineswegs ausschließlich oder auch nur primär auf das von ihnen bearbeitete Sozialsystem, was eine Instrumentalisierung dieser sozialpolitischen Interessen für andere Politikbereiche begünstigte.

Neben den kommunalen Trägern konsolidierten sich die Verbände der freien Wohlfahrtspflege und übernahmen wieder eine im internationalen Vergleich ungewöhnlich große und einflußreiche Rolle bei der Umsetzung öffentlicher Sozialaufgaben, vor allem in Form von Dienstleistungen. Schon in Hinblick darauf, ebenso aber infolge ihrer unangefochtenen fachlichen Kompetenz beanspruchten sie traditionell ein Mitspracherecht bei der rechtlichen Ausgestaltung der Fürsorge. Ihrem christlichen bzw. humanitären Selbstverständnis entsprechend, aber auch im Interesse der Sicherung eigener Domänen übernahmen sie dabei auch anwaltliche Funktionen für die Fürsorgeklientel, die allerdings in den fünfziger Jahren meist ausgeprägt paternalistische Züge trug und auf die individuelle Armutsbekämpfung beschränkt blieb. ${ }^{130}$ Während infolge der Finanzierung dieser Sozialleistung aus Steuermitteln und der Aufgabendurchführung durch Kommunen und Wohlfahrtsverbände die klassischen erwerbswirtschaftlichen Verbände (Gewerkschaften, Arbeitgeber) hier allenfalls gelegentlich eine Rolle spielten, existierte mit dem DV schließlich eine Organisation, die aufgrund ihrer Struktur, der Mechanismen der Interessenkoordination und daraus folgenden halböffentlichen Funktion als geradezu idealtypische Verkörperung eines das deutsche Fürsorgesystem kennzeichnenden „korporatistischen“ Beziehungsgeflechts gedeutet wird. ${ }^{131}$ Einer allgemeineren Definition zufolge wäre von „Korporatismus“ überall dort zu sprechen, „wo die Verbände nicht mehr nur von außen an das politisch-administrative System herantreten, sondern als gleichberechtigte Partner in die Vorbereitung und Ausgestaltung politischer Programme einbezogen sind, und wo sich ein begrenzter Kreis von politisch privilegierten gesellschaftlichen Organisationen wirksam gegenüber dem übrigen Verbändespektrum abzuschotten versteht". ${ }^{132}$ Im Folgenden wird zu untersuchen sein, ob und wann der DV tatsächlich im Sinne eines solchen „korporatistischen Arrangements“133 von staatlichen Stellen und privilegierten Verbänden wirksam wurde, oder ob er nicht vielmehr primär als Diskussionsforum diente und gegebenenfalls mit seinem fachlichen Renommee Entscheidungen stützen sollte, die anderswo gefällt worden waren.

\section{Wer zablt für wen? Die Umverteilung der Fürsorgelasten in den Nachkriegsjabren}

Die Bemühungen der Träger der öffentlichen Fürsorge um finanzielle Konsolidierung hatten eine doppelte Stoßrichtung: Da zum einen das alte Prinzip des "gewöhnlichen Aufenthalts" kaum mehr funktionierte, ging es ihnen um eine Neuordnung der Lastenverteilung der Fürsorgeträger untereinander; gleichzeitig

130 Für heute vgl. ebenda, S. 132ff., 154ff., $265 \mathrm{ff}$.

131 Vgl. ebenda, S. $419 f$.

132 Ebenda, S. 364.

133 Vgl. ebenda, S. 418. 
zielten diese Bemühungen auf eine grundsätzliche finanzielle Sanierung der Kommunalhaushalte durch die (Rück-)Übertragung wesentlicher Fürsorgekosten auf die Länder und später den Bund. Eng verknüpft waren diese Bemühungen mit den hier nicht vertieft zu behandelnden Problemen eines künftigen allgemeinen "Lastenausgleichs" und des Ringens um die Finanzverfassung und damit den Status der Gemeinden im künftigen Bundesstaat.

Die fürsorgerechtliche Lastenverteilung war 1924 in $\$ 7 \mathrm{RFV}$ so geregelt worden, daß grundsätzlich derjenige Bezirksfürsorgeverband zu zahlen hatte, in dem der Hilfsbedürftige „den gewöhnlichen Aufenthalt“, also den „Mittelpunkt seiner Lebensbeziehungen“ und „irgend eine Behausungsmöglichkeit"134 hatte. Wurde jemand in einem anderen Bezirksfürsorgeverband hilfsbedürftig, war dieser Fürsorgeverband des "tatsächlichen Aufenthalts“ zur Fürsorge „vorläufig“ verpflichtet, erhielt aber die Kosten durch den „endgültig“ verpflichteten Fürsorgeverband des gewöhnlichen Aufenthalts ersetzt. War ein solcher gewöhnlicher Aufenthalt nicht vorhanden oder zu ermitteln, hatte der Landesfürsorgeverband die Kosten zu tragen, in dessen Bezirk sich der Betroffene zu Beginn seiner Hilfsbedürftigkeit befand. In jedem Falle sollten also Städte und Landkreise davor bewahrt werden, für vorübergehend Zugezogene die Fürsorgekosten tragen zu müssen. ${ }^{135}$ Streitfälle zwischen einzelnen Fürsorgeverbänden regelten die Verwaltungsgerichte und in letzter Instanz das Bundesamt für Heimatwesen, dessen „kasuistische Rechtsprechung “ 136 bis Ende der dreißiger Jahre 96 Bände füllte. ${ }^{137}$

Eine derartig komplizierte Regelung setzte voraus, daß die Bevölkerung überwiegend seßhaft war und Verwaltung und Geldverkehr reibungslos funktionierten. Das war bereits im Laufe des Krieges immer weniger der Fall, so daß die Reichsregierung sich um eine Vereinfachung bemühte. ${ }^{138}$ Vor allem die „Dritte Verordnung zur Vereinfachung des Fürsorgerechts" vom 11. Mai $19433^{139}$ veränderte das bisherige System gravierend, indem sie die Verpflichtung zum Kostenersatz zwischen den Fürsorgeverbänden für die Dauer des Krieges weitgehend aufhob und damit faktisch zum Prinzip des „tatsächlichen Aufenthalts“ überging. ${ }^{140} 1944$ schließlich wurde die gesamte fürsorgerechtliche Rechtsprechung stillgelegt und die sogenannte außerordentliche Fürsorgelast bei der Anstaltsunterbringung Behinderter, Alkoholkranker u.ä. auf die jeweiligen Landesfürsorge-

134 Muthesius, Grundlagen, S. 35.

135 Darüber hinaus sah die RFV besondere Schutzmaßnahmen für Orte mit Anstalten ( $\left.\int S 8,9 \mathrm{RFV}\right)$ vor.

136 Rundschreiben 1947, S. 7.

$137 \mathrm{Vgl}$. Muthesius, Grundlagen, S. 13.

138 Bereits kurz nach Kriegsbeginn wurden die Befugnisse des Bundesamtes für das Heimatwesen auf das Reichsinnenministerium übertragen und auf wenige grundsätzliche Fälle beschränkt; das Bundesamt wurde aufgelöst; vgl. Verordnung zur Vereinfachung des Fürsorgerechts vom 7.10.1939, RGBl. 1939 I, S. 2002.

139 RGBl. 1943 I, S. 301.

140 Ausgenommen waren u.a. sog. Fälle von „Abschiebung“ (wenn ein Hilfsbedürftiger statt der notwendigen Hilfe nur die Fahrkarte zur Reise in den nächsten Bezirksfürsorgeverband erhielt). 
verbände mit dem Recht auf Kostenerstattung übertragen; da diese weitere Verordnung erst ab dem 1. April 1945 in Kraft trat, wurde sie jedoch nicht mehr überall durchgeführt. ${ }^{141}$

Mit dem Ende des Krieges kam auch der noch verbleibende Lastenausgleich zum Erliegen: Improvisierende örtliche Verwaltungen, zusammengebrochene Post- und Verkehrsverbindungen, das Chaos der hereinbrechenden Flüchtlingsströme, umherreisende Kriegsheimkehrer und wohnungslose Ausgebombte machten die Ermittlung eines „gewöhnlichen Aufenthalts“ und eine individuelle Abrechnung obsolet, zumal viele der ursprünglich zuständigen Fürsorgeverbände in den Ostgebieten ohnehin nicht mehr vorhanden oder zu erreichen waren. Hinzu kam, daß die Besatzungsmächte jeglichen Erstattungsverkehr zwischen den Zonen verboten und die einzelnen Länder innerhalb der Westzonen untereinander ihn übergangsweise ebenfalls suspendierten, bis er Anfang 1947 allmählich zonenintern wieder in Gang kam. ${ }^{142}$ In der sowjetischen Zone ruhten Ersatzansprüche der Fürsorgeverbände untereinander völlig und wurden 1947 auch förmlich abgeschafft. ${ }^{143}$ Gleichzeitig erloschen damit auch alle Erstattungsansprüche gegenüber Fürsorgeverbänden anderer Zonen.

Doch auch in den Westzonen blieben ihrerseits die Vorschriften der Dritten Vereinfachungsverordnung bis auf weiteres eigentlich in Kraft. ${ }^{144}$ Diese unklare Situation mußte diejenigen Bezirksfürsorgeverbände auf die Barrikaden rufen, die viele Flüchtlinge, Ausgebombte und Evakuierte aufgenommen hatten, also vor allem Landkreise, die nach Aufhebung von Räumungs- und Einsatzfamilienunterhalt nun gerne wenigstens gegenüber den in den Westzonen gelegenen Heimatstädten der Ausquartierten Ersatzansprüche geltend gemacht hätten, die vorher das Reich getragen hatte. Ähnlich schwierig war die Lage etwa für Orte, in denen Besatzungstruppen stationiert waren und deshalb offenbar Geschlechtskrankheiten gehäuft auftraten, für Kommunen mit Spezialkliniken oder für solche in Grenzgebieten, in die heimatlose Deutsche aus dem Ausland zurückkehrten. Sie alle empfanden die gegenwärtige Lastenverteilung angesichts zunächst ständig wachsender Fürsorgekosten weder als auf die Dauer finanzierbar noch gerecht.

Die meist stark zerstörten Großstädte sahen sich allerdings ebensowenig zur Übernahme weiterer Belastungen und deren administrativer Bewältigung in der Lage, zumal auch sie nun für Personen aufzukommen hatten, die ehemals von an-

141 „Vierte Verordnung zur Vereinfachung des Fürsorgerechts“ vom 9.11.1944, RGBl. 1944 I, S. 323; vgl. NDV 28 (1948), S. 5.

142 Vgl. Rundschreiben 1947, S.7; NDV 30 (1950), S. 257. Im Januar 1946 blockierte auch der Berliner Magistrat jeglichen Erstattungsverkehr mit auswärtigen Fürsorgeverbänden; vgl. NDV 32 (1952), S. 280.

143 Vgl. „Befehl Nr.92 des Obersten Chefs der SMAD“ vom 22.4.1947 nebst Anlage („Verordnung über Sozialfürsorge für die Bevölkerung in der sowjetischen Besatzungszone Deutschlands“), ZVOBl. 1947 Nr. 5, S. 54; „1.Durchführungsverordnung zur Verordnung über Sozialfürsorge vom 22.4.1947 gemäß Befehl Nr. 92 des Obersten Chefs der SMAD“ vom 2. 9.1947, ebenda, Nr. 19, S. 219.

144 Für ihre Aufhebung hätte es einer formellen Feststellung des Kriegsendes bedurft; vgl. Muthesius, Grundlagen, S. 115, Anm. 1. 
deren Sozialleistungsträgern oder vom Reich unterstützt worden waren. ${ }^{145}$ Eine Anpassung der Lastenausgleichsregelungen an die neue Situation, so ein im Auftrag des hessischen Wohlfahrtsministeriums von Polligkeit erstelltes DV-Gutachten vom Herbst $1946^{146}$, sei daher nur möglich im Rahmen eines neu zu regelnden Finanzausgleichs innerhalb der Länder, in dem übergeordnete Träger, „gewissermaßen als Rechtsnachfolger des Reichs“147, vorläufig die Lasten der Kriegsfolgenhilfe tragen würden, bis stabile Verhältnisse eine Dauerlösung ermöglichen würden. Das Gutachten, das dem Stuttgarter Wohlfahrtsausschuß unterbreitet wurde, forderte daher, daß das jeweilige Land für eine Übergangszeit die kriegsbedingten Fürsorgelasten übernehmen solle. Dabei wandte sich Polligkeit zwar explizit gegen einen Ausgleich über zweckgebundene Dotationen, wie er vor 1924 zwischen Reich und Gemeinden bestanden und Anlaß zu heftiger kommunaler Kritik gegeben hatte; ebenso lehnte er aber im Interesse einheitlicher Leistungsgewährung und gerechter Lastenverteilung einen Ausgleich über das Steueraufkommen ab, was kommunalen Wünschen sicher mehr entgegengekommen wäre. ${ }^{148}$

Die Ausgangslage für einen finanziellen Lastenausgleich war allerdings bis zur Gründung der Bundesrepublik denkbar schwierig, da bis zur Währungsreform die zunehmende Inflation die tatsächlichen Verhältnisse überdeckte, die finanzielle Situation der Kommunen unterschiedlich und die Finanzhoheit in den einzelnen Westzonen verschiedenartig geregelt war. Vor allem die reale Finanzlage der kriegszerstörten Kommunen war desolat, in vielen Landgemeinden hingegen bis zur Währungsreform scheinbar günstig, zumal sie mangels Material und Arbeitskräften auf Investitionen in ihre Infrastruktur verzichten mußten. ${ }^{149}$ Die traditionelle Haupteinnahmequelle der Kommunen, die Grund- und Gewerbesteuern, war infolge der Kriegszerstörungen nur mehr wenig ergiebig. Daneben erhielten die Kommunen bald wieder Zuweisungen aus Reichs- bzw. Landesmitteln, allerdings nicht in Form eines geregelten, den tatsächlichen Erfordernissen entsprechenden Finanzausgleichs, sondern in den Ländern unterschiedlich und nur willkürlich und am ad hoc-Bedarf orientiert. ${ }^{150}$

145 Frankfurt a.M. etwa hätte eigentlich im Frühherbst 1947 noch rund 22000 auswärtig untergebrachte Evakuierte als endgültig verpflichteter Fürsorgeverband zu unterstützen; allein die dafür notwendige Verwaltungsarbeit hätte das städtische Sozialamt kaum bewältigen können; vgl. Sorg, Aufgaben, S. 50.

146 Vgl. Rundschreiben 1946, S.50f. Zur Verfasserschaft siehe Krug von Nidda, Polligkeit, S. 256.

147 Polligkeit, Stand, S. 26.

148 Vgl. Rundschreiben 1946, S. 51.

149 Vgl. für die britische Zone Scherpenberg, Finanzwirtschaft, S. 120ff., 132ff., 168ff.; ferner allgemein Deutschland-Jahrbuch (1949), S. 55; Frank, Finanzen; Unruh, Lage, S. 75f.; für die Landkreise auf der Basis hessischer Daten vom Herbst 1948: Asemann, Landkreise. Als Gemeindeverbände hatten die Landkreise nur ein beschränktes Steuerrecht und finanzierten sich durch Zuweisungen des Landes und Umlagen der kreisangehörigen Gemeinden.

150 Zum Finanzausgleichs-Problem in den Nachkriegsjahren vgl. Scherpenberg, Finanzwirtschaft, S. 142f., 166ff., 375ff.; Habermehl, Finanzausgleich, S. 713ff.; Voigt, Auswirkungen, S.119ff.; Renzsch, Finanzverfassung, S.22ff.; ferner Deutschland-Jahrbuch (1949), S.55f. Zur Organisation der Finanzverwaltung in den drei Westzonen siehe Schweigert, Finanzverwaltung, S. $43 \mathrm{ff}$. 
In der britischen Zone kontrollierte zunächst nur die Militärregierung die öffentlichen Finanzen, und die Gemeinden erhielten anfänglich entsprechend dem Ausgleichssystem von 1944 „erstarrte Zuschüsse“ aus Reichsmitteln, die allerdings die neuen Belastungen kaum auffangen konnten, außerdem von Fall zu Fall vor allem für Fürsorgeausgaben ${ }^{151}$ Zuschüsse oder Kreditgenehmigungen. Ende 1946 übertrug die Militärregierung u.a. die Durchführung des Länderfinanzausgleichs auf ein Zonenhaushaltsamt und die Aufkommenshoheit für eine Reihe bisheriger Reichssteuern auf die Länder, die gleichzeitig den Gemeindefinanzausgleich zu regeln hatten; nach Gründung der Bizone wurde den Ländern schließlich die Steuerverwaltungshoheit übertragen. In der amerikanischen wie in der französischen Zone hingegen übertrugen die Militärregierungen die Finanzhoheit von vornherein auf die Länder. ${ }^{152}$ Doch auch hier kam es nicht zu einem systematischen Ausgleich und einer ausreichenden Ausstattung der Gemeinden mit einem eigenen Steueraufkommen. ${ }^{153}$

Statt dessen suchte man in den Ländern der Westzonen Übergangslösungen zumindest für das vordringliche Problem der Lastenverteilung bei der Kriegsfolgenhilfe. Nachdem Verständigungsversuche für länder- oder zonenübergreifende Regelungen gescheitert waren, veröffentlichte als erste die hessische Regierung im Mai 1946 eine eigene Verordnung, wonach u.a. sämtliche Kosten der öffentlichen Fürsorge für hilfsbedürftige Flüchtlinge den Kommunen durch das Land erstattet wurden. ${ }^{154}$ Indem die Verordnung den Bezirksfürsorgeverband für zuständig erklärte, in dem sich ein solcher Flüchtling tatsächlich aufhielt, hob sie für diese Gruppe bereits die Unterscheidung zwischen vorläufiger und endgültiger Fürsorgepflicht auf und beschritt damit den von den Fürsorgefachleuten vorgeschlagenen, einzig realistischen Reformweg. ${ }^{155}$ Für das Haushaltsjahr 1947 bezog ein Finanzausgleichsgesetz dann auch Evakuierte, Angehörige von Vermißten, Heimkehrer und Kriegsopfer mit ein; von den jeweiligen Fürsorgekosten trug das Land allerdings jetzt nur noch $85 \%$, der unterstützende Bezirksfürsorgeverband 10\% und der zuständige Landesfürsorgeverband 5\%.156 Das entsprach Grundsätzen, die der Gemeinsame Deutsche Finanzrat der Finanzminister der Bizone aufgestellt hatte: Die Landesfürsorgeverbände sollten mit ihrer Quote die Abrechnung

151 Vgl. Scherpenberg, Finanzwirtschaft, S. 194.

152 Vgl. Deutschland-Jahrbuch (1949), S.72f.; Berlin vereinnahmte jetzt sämtliche Reichsund Gemeindesteuern, hatte aber neben den Gemeinde- nun auch sämtliche Staatsaufgaben $\mathrm{zu}$ finanzieren.

153 Vgl. Scherpenberg, Finanzwirtschaft, S. 387f. Mit Bildung der Bizone verbesserten sich die Chancen einer koordinierten Finanzpolitik insofern, als der Wirtschaftsrat für die Länder verbindliche Finanzgesetze erlassen konnte; vgl. auch Deutschland-Jahrbuch (1949), S. 73.

154 „Verordnung zur Regelung des Flüchtlingsdienstes“ vom 23.3.1946, GVBl. für GroßHessen Nr. 13 (10.5.1946), S. 111. Vgl. Rundschreiben 1946, S. 10ff.; Polligkeit, Stand, S. 24; Treibert, Aufgaben.

155 Entsprechend die im Laufe des Jahres 1947 in der US-Zone erlassenen Flüchtlingsgesetze; vgl. NDV 27 (1947), S. 105f.; vgl. allgemein Brackmann/Drilling, Wohlfahrtspflege, S. $77 f$.

156 „Gesetz zur Regelung des Finanzausgleichs für das Haushaltsjahr 1947“ vom 1. 8.1947, GVBl. für das Land Hessen 1947, S. 61; vgl. auch NDV 27 (1947), S. 84f. 
der Kriegsfolgenhilfe übernehmen, die Bezirksfürsorgeverbände zu Sparsamkeit und nicht zuletzt zur schnelleren Integration der Flüchtlinge motiviert werden. ${ }^{157}$ Während innerhalb der amerikanischen und der französischen Zone jeweils unterschiedliche Länderregelungen galten ${ }^{158}$, wurde in der britischen Zone der Finanzausgleich für die Kriegsfolgenhilfe länderübergreifend einheitlich geregelt. Nachdem ab 1. April 1946 die Zonenverwaltung 75\% der Lasten finanziert hatte (Länder 10\%, Bezirksfürsorgeverbände $15 \%$ ), übernahmen später die Länder jeweils $85 \%$ der Kosten der Kriegsfolgenhilfe und gaben den Bezirksfürsorgeverbänden auch für die verbleibenden $15 \%$ eine pauschale Finanzzuweisung. ${ }^{159}$

In der britischen Zone war damit der Weg frei für den Übergang zum Prinzip des tatsächlichen Aufenthalts im Fürsorgelastenausgleich. Polligkeit und andere machten dafür vor allem die bekannten praktischen Gründe geltend ${ }^{160}$, doch die Geschäftsstelle des DV stellte Anfang 1947 auch grundsätzlichere Überlegungen an ${ }^{161}$ : Die Abkehr vom Prinzip des "gewöhnlichen Aufenthalts" sei nur die logische Konsequenz aus der rechtlichen Entwicklung seit 1939. Vor allem bedeute er aber eine wesentliche Verwaltungsvereinfachung auch in normalen Zeiten, denn aufwendige Ermittlungen entfielen ebenso wie viele Rechtsstreitigkeiten und Geldtransaktionen. Darüber hinaus habe in der Vergangenheit die Einzelfall-Abrechnung dazu verleitet, die Kosten möglichst auf einen anderen Träger abzuwälzen: „Diesem Anreiz [...] unterlagen viele Fürsorgesachbearbeiter, manche darunter so stark, daß die Lastenverteilung ihnen wichtiger wurde als Art und Maß der Fürsorgegewährung." Tatsächlich aber hätten zahlreiche Fürsorgeverbände feststellen müssen, daß ihre Einnahmen aus und ihre Ausgaben für Erstattungen längerfristig einander aufgehoben hatten, und daher seinerzeit in Ostpreußen bereits auf die Durchführung der Lastenverteilung verzichtet.

Auch der Vorstand des DST sprach sich im Mai 1947 für einen - vorübergehenden - Übergang zum Prinzip des tatsächlichen Aufenthalts aus, vorausgesetzt, die Länder trügen die Kriegsfolgenhilfe. ${ }^{162}$ Die Stadt- und Landkreise sollten allerdings quotenmäßig an den Kosten beteiligt bleiben: Zum einen sicherte dies gewisse kommunale Mitspracherechte, zum anderen konnte dadurch verhindert werden, daß einzelne Kommunen mit den Landesmitteln allzu freigiebig verfuhren und somit Präzedenzien auch für die reguläre Fürsorge entstanden. Wie das DVGutachten machten die Städtevertreter dabei deutlich, daß das geltende Fürsorgerecht zunächst unverändert beizubehalten und die geforderte Modifikation auf

157 Vgl. Sorg, Aufgaben, S. 52.

158 Vgl. Habermehl, Finanzausgleich, S. 725.

159 Vgl. ebenda, S. 726; NDV 28 (1948), S. 4, ferner das Referat Moning auf der Hauptausschußtagung des DLT am 8./9.10.1948, in: Die Selbstverwaltung 2 (1948), S. 159. Daneben bestand in der britischen Zone bis zum 1.4.1949 ein gesonderter EvakuiertenLastenausgleich, wonach Hamburg und Nordrhein-Westfalen Ausgleichszahlungen an Schleswig-Holstein und Niedersachsen entrichten mußten; vgl. Fürsorgerechtsvereinbarung, S. 40.

160 Vgl. u.a. Polligkeit, Stand, S. 27; ders. in: Rundschreiben 1946, S.22f., 37; Sorg, Aufgaben; Referat des Hamburger Senatsdirektors Völcker, in: NDV 28 (1948), S. 66.

161 Vgl. Rundschreiben 1947, S. 7f.

162 Vgl. NDV 27 (1947), S. 62. 
dem Wege der freien Vereinbarung zwischen den Fürsorgeträgern zu bewerkstelligen sei. Die Neuregelung der Lastenverteilung sollte also kein Einfallstor für weitergehende Reformabsichten werden.

Tatsächlich hatte die Arbeitsgemeinschaft der Fürsorgedezernenten der britischen Zone im Herbst 1947 die Vorarbeiten für ein solches Übereinkommen beendet und beschloß am 18. September 1947 in Steinhude die sogenannte Fürsorgerechtsvereinbarung (FRV), die ab Oktober gelten sollte. ${ }^{163}$ Kernpunkt war die weitgehende Abschaffung der individuellen Kostenerstattung der Bezirksfürsorgeverbände untereinander: „Jeder Hilfsbedürftige muß von dem Bezirksfürsorgeverband unterstützt werden, in dessen Bereich er sich befindet“ (Ziff.1). ${ }^{164}$ Ähnlich wie 1943/44 waren allerdings gewisse Ausnahmen vorgesehen, u.a. für Orte mit Fürsorge- oder Erziehungsanstalten, Kliniken etc. Damit sollte jedoch nicht nur eine ungerechte Belastung vor allem der Anstaltsorte vermieden werden, vielmehr werde „mit dieser Lösung einer späteren, unabweislichen Gesamtreform des Fürsorgerechts nicht annähernd so stark vorgegriffen wie bei einer unterschiedslosen Beseitigung der vorläufigen und endgültigen Fürsorgepflicht“; es gelte, „das wenigstens formell noch bestehende einheitliche Fürsorgerecht nicht inhaltlich so zu verändern, daß bei einer künftigen Gesamtreform durch vorweggenommene grundlegende Änderungen die Erhaltung der Einheitlichkeit erschwert oder gefährdet werden könnte“. ${ }^{165}$ Für verbleibende Streitfälle war eine außergerichtliche Regelung durch regionale Spruchstellen vorgesehen.

Der entscheidende Fortschritt lag nicht in einer Änderung der realen finanziellen Verhältnisse der öffentlichen Fürsorgeträger. Wesentlich war vielmehr, daß die FRV die Lastenverteilung der Fürsorgeverbände untereinander erheblich vereinfachte und wieder „zu klaren Rechtsverhältnissen“166 führte. Das war jedoch nur möglich dadurch, daß die Vereinbarung - anders als die Vereinfachungsverordnung von 1943 - in der britischen Zone in einen länderübergreifend vereinheitlichten Lastenausgleich für die Kriegsfolgenhilfe eingebettet war. So traten auch bis Ende 1947 fast alle Stadt-, vier Fünftel der Landkreise sowie alle Landesfürsorgeverbände dieser Zone der neuen Regelung bei, außerdem die der amerikanischen Zone angehörenden Stadtkreise Bremen und Bremerhaven.

Die ebenfalls von den Fürsorgeverbänden und dem DV gewünschte Verlagerung kostspieliger und überörtlicher Aufgaben auf die Landesfürsorgeverbände ließ sich wegen unterschiedlicher Länderregelungen vorerst nicht verwirklichen. ${ }^{167}$ Vordringliches Nahziel blieb daher die Ausdehnung der FRV auch auf die süddeutschen Länder zumindest der US-Zone. Das wurde allerdings wegen der Unterschiedlichkeit der dortigen Regelungen der Kriegsfolgenhilfe erschwert; hinzu kam, daß die britische Militärregierung noch Anfang 1949 am Erstattungs-

163 Vgl. NDV 28 (1948), S.3ff. (FRV auszugsweise zitiert); NDV 29 (1949), S.90; Sorg, Aufgaben, S. 50f. FRV in der Fassung vom 3.5.1949 in: Fürsorgerechtsvereinbarung.

164 NDV 28 (1948), S. 4.

165 Die Allgemeine Begründung ist abgedruckt in: Fürsorgerechtsvereinbarung, S. 5-7, hier S.7.

166 Vgl. Brackmann/Drilling, Wohlfahrtspflege, S. 18f.

167 Vgl. NDV 28 (1948), S. $61 \mathrm{ff} . ; 30$ (1950), S. $158 \mathrm{ff}$. 
verbot gegenüber anderen Zonen festhielt. 168 Nachdem auch in Bayern die Kriegsfolgenhilfe zu $85 \%$ auf das Land übertragen worden war ${ }^{169}$, übernahmen als erstes die dortigen Fürsorgeverbände zum 1. Oktober 1948 wenig geändert die Bestimmungen der FRV, allerdings nur für ihre Beziehungen untereinander und nicht für den Rechtsverkehr mit außerbayerischen Fürsorgeverbänden. ${ }^{170}$ Im Mai 1949 berieten dann Fürsorgevertreter der britischen und der amerikanischen Zone in Weinheim/Bergstraße über eine künftige gemeinsame Vereinbarung. Ein unter Leitung des damaligen DST-Beigeordneten Hans Muthesius gegründeter „Weinheimer Arbeitskreis“ befürwortete eine nur wenig geänderte Neufassung der FRV und erarbeitete ein Rahmenabkommen, wonach die neu gefaßte FRV von den Fürsorgeverbänden länder- und zonenübergreifend auf unbestimmte Zeit geschlossen und ab dem 1. Juli 1949 angewendet werden sollte. ${ }^{171}$ Außerdem wurde den Fürsorgeverbänden der französischen Zone und West-Berlins der Beitritt ermöglicht, nicht jedoch denjenigen der sowjetischen Zone wegen des dortigen grundsätzlichen Erstattungsverbots. ${ }^{172}$ Ursprünglich hatte der DLT heftigen Widerstand gegen die Einbeziehung der Evakuierten in die neue Ausgleichsregelung geleistet, doch mit der Verabschiedung des Grundgesetzes und der verfassungsmäßigen Überführung der Kriegsfolgelasten auf den Bund verlor das Evakuiertenproblem an finanziellem Gewicht.

Anfang 1950 waren bereits sämtliche Landes- sowie die weit überwiegende Zahl der Bezirksfürsorgeverbände der ehemaligen Bizone der FRV nebst Rahmenabkommen beigetreten. ${ }^{173}$ Die Länder der französischen Zone hingegen hatten den Erstattungsverkehr im Laufe der Jahre ganz unterschiedlich geregelt und teilweise wieder auf die Weimarer Vorschriften zurückgegriffen, was zu erheblichen Komplikationen in den Beziehungen mit den anderen Ländern führte. Doch im Laufe des Jahres 1950 wurden auch die dortigen Fürsorgeverbände mit wenigen Ausnahmen einbezogen, so daß der DV im November melden konnte: „Das Ziel, [...] durch eine freiwillige Vereinbarung der Fürsorgeverbände das geltende Fürsorgerecht den veränderten Verhältnissen anzupassen und damit eine besonders dringende Teilreform des Fürsorgerechts vorwegzunehmen und in der Praxis zu erproben, ist nunmehr im wesentlichen erreicht." 174 Nachdem die Finanzbeziehungen zwischen Berlin und dem Bund geregelt worden waren, trat auch Berlin Ende Juni 1952 der FRV bei. ${ }^{175}$ Die Vereinbarung blieb bis zur Verab-

168 Vgl. NDV 29 (1949), S. 90.

169 Vgl. Die Selbstverwaltung 4 (1950), S. 279.

170 Vgl. auch für das Folgende, NDV 29 (1949), S. 89ff.; 125; Referat Schmerbeck, in: Die Selbstverwaltung 3 (1949), S. 253f.; Keese, 25 Jahre.

171 Neufassung der FRV und Rahmenabkommen sind abgedruckt in: NDV 29 (1949), S.125ff. (Berichtigung: S. 180). Zur Erläuterung siehe ausführlich Fürsorgerechtsvereinbarung.

172 Mit dem Inkrafttreten der neuen FRV am 1.7.1949 wurde gleichzeitig der Erstattungsverkehr zwischen den Verbänden der britischen und der amerikanischen Zone wieder genehmigt; vgl. Otto, Fürsorge, S. 55.

173 Vgl. NDV 30 (1950), S. 13f., $256 f$.

174 Ebenda, S. 257.

175 Vgl. NDV 32 (1952), S.280. Das Saarland, das ab 1950 wieder uneingeschränkt zum Prinzip des gewöhnlichen Aufenthalts zurückgekehrt war und gleichzeitig den Erstat- 
schiedung des BSHG unverändert bestehen; das BSHG übernahm dann die meisten ihrer materiellen Bestimmungen, bis die FRV 1965 schließlich mit dem Kernstück des schiedsgerichtlichen Verfahrens neu gefaßt wurde und am 1. Januar 1966 in Kraft trat. ${ }^{176}$

Der tatsächliche Aufenthalt konnte als Leitprinzip der fürsorgerechtlichen Lastenverteilung zu diesem Zeitpunkt allerdings nur durchgesetzt werden, weil parallel der Finanzausgleich der Kriegsfolgenhilfe auf Landes-, dann vor allem auf überzonaler Ebene geregelt wurde. Nach entsprechenden Vorarbeiten unter Leitung Polligkeits erarbeitete ein Arbeitsstab, dem er ebenfalls angehörte, im Auftrag der neuen Verwaltung für Finanzen des Vereinigten Wirtschaftsgebiets im Winter 1947/48 Vorschläge für einen interzonalen Lastenausgleich für die öffentliche Fürsorge: Danach sollten die Fürsorgeaufwendungen für Vertriebene, Zugewanderte aus der SBZ und aus Berlin sowie für Evakuierte ausgeglichen werden. Abgerechnet werden sollten Hilfen auf der Grundlage der RFV und von Sonderbestimmungen des Fürsorge- und Gesundheitswesens. Neben diesen Individualkosten sollten auch die allgemeinen Kosten für den Transport und die Unterbringung in Lagern erstattungsfähig sein. ${ }^{177}$

Strittig blieb hingegen, wie die Belastungen der Länder und damit ein Schlüssel für die Ausgleichszahlungen zu ermitteln seien, da der Ausschuß Zahlungen auf der Basis von Durchschnittssätzen favorisierte. Ein Gutachten des DV vom April 1948 sah schließlich Schleswig-Holstein, Niedersachsen, Bayern und Hessen als Empfänger, Nordrhein-Westfalen, Württemberg-Baden und die beiden Stadtstaaten als Zahler in einem künftigen Lastenausgleich vor. Während die Empfängerländer dem vorgeschlagenen Ausgleichsschlüssel wenige Tage vor der Währungsreform zustimmten, legten die Geberländer Protest ein und forderten die Berücksichtigung der Kosten für die Besatzung oder für Kohle- und Stahlsubventionen. Auch auf einer Sitzung der Länderfinanzminister Anfang Juli konnte keine Einigkeit erzielt werden. Dabei bestand gerade jetzt, kurz nach der Währungsreform, in den Augen führender Kommunalvertreter dringender Handlungsbedarf, rechnete man doch allerorten mit einem erheblichen Anstieg der Fürsorgekosten. ${ }^{178}$

tungsverkehr mit anderen Fürsorgeverbänden fast völlig unterbunden hatte, vgl. NDV 31 (1951), S. 212f., folgte schließlich zum 1.7.1957; vgl. Keese, 25 Jahre, S. 75.

176 Vgl. Keese, 25 Jahre, S.75f. Ergänzt wurde die FRV durch das „Kasseler Abkommen“ vom 29.4.1952, abgedruckt in: NDV 32 (1952), S.163f., einer Sondervereinbarung der Landesfürsorgeverbände, die zur finanziellen Entlastung von Orten mit Entbindungsanstalten die Zuständigkeiten modifizierte; ferner durch die „Bonner Vereinbarung“ vom 2.9.1952 (Anlage zum RdSchr. des BMI vom 4.11.1952, GMBl. S. 305) und die „Freiburger Ergänzungsvereinbarung“ vom 30.7.1953 (Anlage zum RdSchr. des BMI vom 19.2.1954, GMBl. S.91), die zur Entlastung der Grenzfürsorgeverbände die Zuständigkeit für hilfsbedürftige Deutsche im Ausland bzw. Rückwanderer und Ausländer regelten.

177 Zur Entwicklung der bizonalen Finanzverwaltung vgl. Schweigert, Finanzverwaltung, S. 93ff.; zum Fürsorgelastenausgleich Krug von Nidda, Polligkeit, S.269ff.; NDV 28 (1948), S. 68, $123 f$.; 36 (1956), S. $149 f f$.

178 Vgl. Blaum, Geldneuordnung, S. 94. Ferner einen Bericht Polligkeits für die amerikanische Militärregierung über die Auswirkungen der Währungsreform auf die deutsche Wohlfahrtspflege vom 9.7.1948, in: NDV 28 (1948), S. $116 f$. 
Tatsächlich stieg ja im Laufe des Herbstes die Zahl der Fürsorgeempfänger vorübergehend wieder deutlich an, jetzt ergänzt um die neue Gruppe der „Währungsgeschädigten“, während die z.T. beträchtlichen kommunalen Altgeldguthaben annulliert worden waren und vor allem die Landkreise trotz Erstausstattung bald erhebliche Haushaltsdefizite aufwiesen. ${ }^{179}$ Allein in der Bizone kostete nur die offene Fürsorge für Empfänger der Kriegsfolgenhilfe in der Zeit von Oktober 1948 bis Ende März 1949 rund 376 Mio. DM. ${ }^{180}$ Wollte man nicht länger hinnehmen, daß aufgrund der unterschiedlichen Steuerkraft und finanziellen Leistungsfähigkeit der Länder und ihrer Fürsorgeverbände letzten Endes die Hilfsbedürftigen unterschiedlich und oftmals nicht ausreichend unterstützt wurden, war ein länderübergreifender Ausgleich das Gebot der Stunde. Für das Rechnungsjahr 1948 einigten sich die Länder des Vereinigten Wirtschaftsgebiets auf eine - nur ungenügende - Finanzhilfe von knapp 87 Mio. DM zumindest für das leistungsschwächste Land, Schleswig-Holstein. Doch erst Anfang August 1949 kam es nach schwierigen Verhandlungen zu einem Gesetz des Wirtschaftsrates, das den horizontalen Länderausgleich der Kriegsfolgelasten für die erste Hälfte des Rechnungsjahres 1949 in der Bizone vorläufig regelte; für das zweite Halbjahr wurde auch Rheinland-Pfalz einbezogen. ${ }^{181}$

Eine Lösung in größerem Rahmen ermöglichte erst die Gründung der Bundesrepublik. Das Grundgesetz bestimmte in Art. 120 Abs. 1, daß der Bund neben den Aufwendungen für die Besatzungskosten auch „die sonstigen inneren und äußeren Kriegsfolgelasten nach näherer Bestimmung eines Bundesgesetzes“ trug, und schuf damit die Voraussetzungen für einen vertikalen Ausgleich zwischen Bund und Ländern auch bei der Kriegsfolgenhilfe. ${ }^{182}$ Erleichtert wurde diese Lastenübertragung auf den Bund dadurch, daß seit Herbst 1949 immer mehr Kriegsfolgenhilfeempfänger aus Abgaben finanzierte Leistungen nach dem neuen „Soforthilfegesetz" erhielten und aus der Fürsorge ausschieden.

An der praktischen Umsetzung des Art. 120 GG arbeitete Polligkeit nun wieder an prominenter Stelle mit: Unter seiner Leitung verfaßte ein „Arbeitsstab für Fragen der Kriegsfolgenhilfe" 183 im Auftrag des von den Ministerpräsidenten der Länder eingesetzten Ausschusses für den Finanzausgleich im Herbst 1949 einen

179 Vgl. für Nordrhein-Westfalen: Die Selbstverwaltung 2 (1948), S. 158f.; für Hessen Asemann, Landkreise.

180 Vgl. NDV 29 (1949), S. 202.

181 „Gesetz zur vorläufigen Regelung der Kriegsfolgelasten im Rechnungsjahr 1949“ vom 6. 8.1949, WiGbl., S. 235, „Gesetz zur Regelung von Kriegsfolgelasten im 2. Rechnungshalbjahr 1949“ vom 21.3.1950, BGBl. 1950, S. 43. Vgl. Deutschland-Jahrbuch 1953, S. 205; Voigt, Auswirkungen, S. 122; Renzsch, Finanzverfassung, S. 28ff., 33ff., $48 \mathrm{ff}$.

182 Außerdem ermächtigte Art. 106 Abs. 4 GG den Bund, mit Zustimmung des Bundesrates einen horizontalen Finanzausgleich zwischen den Ländern durchzuführen. Statt dessen bildeten die Länder jedoch einen eigenen Ausgleichsstock, aus dem nach einem bestimmten Schlüssel horizontale Ausgleichszahlungen zwischen den steuerstarken und den steuerschwachen Ländern geleistet wurden; vgl. Voigt, Auswirkungen, S.134f.

183 Stellvertretender Vorsitzender dieses aus rund zwanzig Fürsorge- und Finanzexperten bestehenden Gremiums war Hans Muthesius; zu den Mitgliedern gehörte außerdem u.a. der spätere Leiter der Sozialabteilung des Bundesinnenministeriums (BMI), Wilhelm Kitz; vgl. NDV 30 (1950), S. 57; Heisig, Armenpolitik, 1990, S. 551. 
entsprechenden Richtlinienentwurf für das Rechnungsjahr 1950.184 Auch jetzt ging es wieder darum, „das vielfältig verästelte und regional sehr differenzierte Gebiet der Kriegsfolgenhilfe so zu vereinheitlichen, daß der Gesamtkomplex der kriegsverursachten Fürsorgelasten als eine in sich geschlossene und eindeutig bestimmbare Lastengruppe aus den Länderhaushalten herausgelöst und auf den Bundeshaushalt übergeleitet werden konnte“185.

Bei der Bestimmung des Personenkreises knüpfte der Entwurf an die Vorschläge des ehemaligen Arbeitsstabes der Finanzverwaltung an, erweiterte ihn jedoch um Ausländer und Staatenlose, die Angehörigen von Vermißten, sämtliche Kriegsheimkehrer, die Kriegsopfer beider Weltkriege und ihnen Gleichgestellte sowie die illegalen Grenzwanderer aus der SBZ/DDR. Dabei erwies sich die praktische Ermittlung der Kosten der individuellen Fürsorge abermals als problematisch. Nach den schlechten Erfahrungen mit Durchschnittssätzen empfahl der Entwurf, den Ländern und Kommunen die tatsächlichen Aufwendungen zu ersetzen, besonders da mittlerweile die Begriffsmerkmale der Fürsorgekosten für Buchungen und Statistik vereinheitlicht waren. Wie vom Finanzausschuß der Ministerpräsidentenkonferenz selbst vorgeschlagen, sollte der Bund nur $85 \%$ der Aufwendungen für die Kriegsfolgenhilfe übernehmen, so daß den Ländern eine „Interessenquote“ von 15\% verblieb, die sie ihrerseits wieder auf die Fürsorgeverbände umlegen konnten. Die quotenmäßige Beteiligung der Länder war notwendig, da bereits deutlich wurde, daß die dem Bund zugewiesenen Steuern zur Deckung der Ausgaben nicht genügten. ${ }^{186}$ Außerdem sollte die „Interessenquote“ den sparsamen Umgang mit den Bundesmitteln fördern. ${ }^{187}$ Als zusätzliche Sicherung des Bundes gegen eine „unberechtigte Erhöhung der Richtsätze“ durch einzelne Länder sah der Arbeitsstab ferner vor, daß „Richtsätze und Unterstützungsrichtlinien in der Kriegsfolgenhilfe keine anderen sein dürfen als in der allgemeinen Fürsorge“188. Erstattet werden sollten nur die gesetzlichen Pflichtleistungen nach den ortsüblichen Richtsätzen sowie Leistungen, die aufgrund von Sonderbestimmungen des Fürsorge- und Gesundheitswesens gewährt wurden, wie die Tuberkulosehilfe, die Geschlechtskrankenfürsorge und Leistungen für Kriegsblinde und Hirnverletzte. Mit dieser Bindung der Kriegsfolgenhilfe an Richtsätze und Leistungen der allgemeinen Fürsorge wollte der Arbeitsstab allen Bestrebungen nach einer Wiederbelebung der Gruppenfürsorge einen festen Riegel vorschieben und folgte damit der generellen Linie des DV und insbesondere Polligkeits.

$184 \mathrm{Vgl}$. NDV 30 (1950), S. $5 \mathrm{ff}$.

185 So der damals im Hauptreferat für Finanzen des Länderrats tätige und spätere Ministerialrat im Bundesfinanzministerium (BMF) Herbert Fischer-Menshausen in: NDV 31 (1951), S. 130.

186 Vgl. NDV 34 (1954), S. 258.

187 Vgl. auch Renzsch, Finanzverfassung, S. 75ff. Tatsächlich zog man mit den Interessenquoten letzten Endes die Konsequenzen aus den Erfahrungen nach dem Ersten Weltkrieg. Nach der Erzbergerschen Finanzreform von 1920 war das Dotationssystem für die Sonderfürsorgebereiche der Kriegs- und der Inflationsopfer vom Reichsarbeitsministerium und den kommunalen Spitzenverbänden u.a. deshalb heftig kritisiert worden, weil die Trennung von Aufgaben und Mittelverwendung allseits zu deren Verschwendung geführt habe; vgl. Sachße/Tennstedt, Geschichte, Bd. 2, S. $176 f$.

188 Begründung zum Entwurf, in: NDV 30 (1950), S. 10. 
Nachdem der Entwurf dem Bundesrat Anfang Januar 1950 übergeben worden war, bildete er die Grundlage für die vorläufigen Richtlinien des Bundesinnenministeriums und des Bundesministeriums der Finanzen über Kriegsfolgenhilfe vom 17. März 1950 (Abrechnungserlaß) ${ }^{189}$ und später für die entsprechenden Teile des Ersten Überleitungsgesetzes vom 28. November 1950190, das im Sinne des Art. 120 GG die Lasten der Kriegsfolgenhilfe sowie weitere Soziallasten und überregionale Aufgaben rückwirkend ab 1. April 1950 von den Ländern auf den Bund übertrug. Gleichzeitig flossen nun auch die meisten der bisher den Ländern zustehenden Verbrauchssteuern sowie die Umsatz- und die Beförderungssteuer dem Bund zu, die zusammen mit der „Interessenquote“ der Länder die Ausgaben der Kriegsfolgenhilfe etc. ungefähr decken sollten. ${ }^{191}$ Das Überleitungsgesetz folgte damit weitgehend den Vorschlägen des Arbeitsstabes, erhöhte allerdings die „Interessenquote“ der Länder auf 25\%. Da diese Quote auf die Gemeinden und Gemeindeverbände umgelegt werden durfte, führte das Erste Überleitungsgesetz für die Fürsorgeverbände gegenüber den bisherigen Länderregelungen nicht immer zu einer finanziellen Entlastung; außerdem benachteiligte das Quotensystem auch weiterhin die steuerschwachen Flüchtlingsländer. Die Kriegsfolgenhilfe-Regelungen wurden daher in den Folgejahren wiederholt auf Bundesebene geändert, verloren aber im Zuge des allgemeinen wirtschaftlichen Aufschwungs und infolge der Sozialgesetzgebung zugunsten der Vertriebenen, Kriegsopfer etc. allmählich ohnehin an Brisanz.

Neben der erhöhten Quote enthielt das Erste Überleitungsgesetz aus Ländersicht eine weitere Kautel, die um der finanziellen Entlastung willen hinzunehmen war: Änderungen des Landesrechts zur Kriegsfolgenhilfe waren künftig, „wenn sie von grundsätzlicher Bedeutung oder von erheblicher finanzieller Auswirkung für den Bund sind, [von] der Zustimmung der zuständigen Bundesorgane" ( $\int 4$ Abs. 2) abhängig. Da für die Kriegsfolgenhilfe das allgemeine Fürsorgerecht maßgeblich war, könnten theoretisch also die Bundesministerien des Innern und der Finanzen ein gewichtiges Wort etwa bei Anrechnungsvorschriften oder Richtsätzen mitreden. „Die hohe finanzielle Beteiligung des Bundes an den Lasten der öffentlichen Fürsorge“, kommentierte dann auch der DV-Nachrichtendienst im Januar 1951, „begründet eine Einflußnahme des Bundes auf die Handhabung der Fürsorge durch die Fürsorgeverbände, die im Bundesfürsorgerecht sonst nicht vorgesehen ist“. ${ }^{192}$ Die praktischen Auswirkungen dieser Vorschrift bedürften einer gesonderten Untersuchung. Aus Sicht der Bundesregierung vier Jahre später hatten sie jedenfalls die „Gefahr einer ungleichmäßigen und unwirtschaftlichen Verwendung der Bundesmittel“ nicht bannen können. ${ }^{193}$

Aus Sicht der Verfechter des Fürsorgeprinzips bot das Erste Überleitungsgesetz nichtsdestoweniger neben dem unmittelbaren finanziellen einen zumindest systematischen Vorteil: Die Bundesfinanzierung der Kriegsfolgenhilfe garantierte vor-

189 Vgl. ebenda, S. 57 ff., 80.

190 „Erstes Gesetz zur Überleitung von Lasten und Deckungsmitteln auf den Bund (Erstes Überleitungsgesetz)“ vom 28.11.1950, BGBl. 1950, S.773. Vgl. NDV 31 (1951), S. 2 ff.

191 Vgl. Deutschland-Jahrbuch (1953), S. 202.

192 NDV 31 (1951), S. 3.

193 Vgl. die Begründung der Bundesregierung u.a. zum Vierten Überleitungsgesetz, auszugsweise wiedergegeben in: NDV 32 (1952), S.257ff., hier S. 259. 
erst den Bestand der hergebrachten öffentlichen Fürsorge (als Einheitsfürsorge), indem sie deren Maßnahmen für eine neue Klientel finanzierte, ohne ihre rechtlichen und organisatorischen Grundlagen anzutasten oder ihre Ausführung zunächst detaillierter zu regeln.

\section{Für die (Wieder-)Vereinheitlichung des Fürsorgerechts}

Schon bald nach Kriegsende schufen die amerikanische und die britische Militärregierung fürsorgerechtliche Fakten, die für die weitere Entwicklung der Fürsorge von nachhaltiger Bedeutung sein sollten: Ohne das bisherige Recht förmlich aufzuheben, verboten sie, einem Kontrollratsbeschluß entsprechend, im August bzw. November 1945 die Anwendung der reichsrechtlichen Vorschriften über die gehobene Fürsorge. Formal unklar blieb zwar, ob damit nur die höheren Richtsätze verboten waren, wie es die britischen finanztechnischen Anweisungen nahelegten, oder auch weitergehende Privilegien und sozialfürsorgerische Maßnahmen, zumal die amerikanische Anordnung von "elimination of special assistance programs“ sprach. ${ }^{194}$ In der Praxis wurden die Vorschriften der RGr. über die gehobene Fürsorge vorerst offensichtlich kaum noch angewendet ${ }^{195}$, so daß de facto die Einheitsfürsorge in den beiden Besatzungszonen weitgehend durchgesetzt war (Freibeträge für Renten durften in der britischen Zone allerdings weiter gelten). Parallel dazu wurden die Richtsätze auf britische Anordnung hin auf dem Stand vom 1. Oktober 1945 festgeschrieben und erst im Zuge der Bildung der Länderparlamente 1946/47 wieder freigegeben. ${ }^{196}$ Innerhalb der französischen Zone war die Entwicklung unterschiedlich: Während in Teilen von Rheinland-Pfalz und im Kreis Lindau die Einheitsfürsorge eingeführt wurde, galten bis 1949 in Baden und Württemberg-Hohenzollern noch immer unterschiedliche Richtsätze für allgemeine und gehobene Fürsorge. ${ }^{197}$ Im Saarland schließlich wurde die gehobene

194 Die amerikanische Militärregierung hielt sich damit zunächst streng an die Vorgaben der bekannten Besatzungsdirektive JCS 1067 vom April 1945, die nur dazu ermächtigte, das Sozialrecht zu entnazifizieren und „to direct German authorities to maintain or re-establish nondiscriminatory systems of social insurance and poor relief“ (Ziff. 25); Direktive abgedruckt in: Cornides/Volle, Frieden, S. 58-73, hier S. 66. Die genannte und die im nächsten Jahr folgenden Anordnungen wurden fast alle nicht veröffentlicht. Es handelte sich dabei um die Weisung des Hauptquartiers der amerikanischen Streitkräfte in Europa vom 4.8.1945 betr. die Kontrolle der deutschen öffentlichen Fürsorge- und Unterstützungspolitik (AG 091, 4 GEC - AGO), in der britischen Zone um die Finanztechnische Anweisung Nr. 54 vom 5.11.1945, die Finanztechnische Anweisung Nr. 70 vom 10.4.1946 (Abschriften von den deutschen Übersetzungen der amerikanischen bzw. britischen Anweisungen in: LAB, B Rep. 142-9, 1291) sowie die Verordnung Nr. 99 betr. Verbotene Ausgaben vom 15.9.1947, Ziff. 22 des Anhangs, Amtsblatt der Militärregierung Deutschland. Britisches Kontrollgebiet No.21, S. 589. Vgl., auch für das Folgende, NDV 30 (1950), S.255f. (Zitat ebenda, S.256); Brackmann/Drilling, Wohlfahrtspflege, S. 25, 92. Vgl. ferner Willing, Vorgeschichte, S. $598 \mathrm{ff}$.

195 Vgl. Muthesius, Grundlagen, S.17, 100; NDV 27 (1947), S.73f.; Brackmann/Drilling, Wohlfahrtspflege, S. 92.

196 Vgl. Finanztechnische Anweisung Nr. 54, LAB, B Rep. 142-9, 1291.

197 Vgl. NDV 29 (1949), S. 6f. Im Herbst 1950 gab es auch in diesen Ländern die Einheitsfürsorge, vgl. NDV 30 (1950), S. 255. 
Fürsorge durch die Landesregierung bereits Anfang 1948 auch formell abgeschafft. 198

Die Sistierung der Gruppenfürsorge sollte primär Ausgaben vermindern; darüber hinaus schien die Privilegierung einzelner Gruppen den Grundsätzen eines demokratischen Neuaufbaus zu widersprechen ${ }^{199}$, zumal die gehobene Fürsorge während der Zeit des Nationalsozialismus immer mehr zu „eine[r] Art Ehrensold für leistungs- und artbewußte ,Volksgenossen“" 200 umfunktioniert worden war. Im Nachrichtendienst des DV wurde die alliierte Maßnahme als Erfüllung einer alten Forderung begrüßt ${ }^{201}$ und als logische Konsequenz der realen Entwicklungen in ihrer Bedeutung öffentlich eher bagatellisiert. ${ }^{202}$ Dabei bedeuteten diese Verbote langfristig eine sozialpolitische Richtungsänderung: Anders als während und nach dem Ersten Weltkrieg, als neue Gruppen von Hilfsbedürftigen durch neue, privilegierte Fürsorgebereiche in dieses Sicherungssystem integriert werden sollten, wurde jetzt eine fürsorgerische Sonderbehandlung gerade auch der neuen Gruppe der Flüchtlinge ausgeschlossen. Auch die fürsorgepolitischen Weichen waren damit letzten Endes - obwohl von den Verfechtern der Einheitsfürsorge so gar nicht gewollt - in Richtung einer Überführung dieser neuen Gruppen in andere Sicherungssysteme gestellt. ${ }^{203}$

Darüber hinaus gab es im Alliierten Kontrollrat im Herbst 1945 offensichtlich von sowjetischer Seite initiierte Pläne, im Rahmen des Umbaus des gesamten deutschen Sozialleistungssystems „eine Gesamtreform zur Vereinfachung und Vereinheitlichung des deutschen Fürsorgerechtes in die Wege zu leiten“. ${ }^{204} \mathrm{Da}$

198 Vgl. Zweite Anordnung über Ergänzung und Änderung fürsorgerechtlicher Vorschriften vom 2.3.1948, Amtsblatt des Saarlandes, S.311; NDV 31 (1951), S. 212f.

$199 \mathrm{Zu}$ den Motiven der Besatzungsmächte vgl. aus deutscher Sicht u.a. Jellinghaus, Betrachtungen zur Anwendbarkeit, S.7; NDV 33 (1953), S. 255; Krug von Nidda, Polligkeit, S. 257.

200 Sachße/Tennstedt, Geschichte, Bd.3, S. 274.

201 In den zwanziger Jahren hatte der DV unter Polligkeit die Gruppenfürsorge massiv bekämpft, allerdings nicht im Interesse einer nicht schichtenspezifischen Fürsorge, sondern um unter dem Banner des Individualprinzips den kommunalen Trägern ihre Gestaltungsmacht bei der individuellen Fürsorgebemessung zu erhalten; vgl. Leibfried, Existenzminimum, S. 497ff.; Sachße/Tennstedt, Geschichte, Bd. 2, S. $173 \mathrm{f}$.

202 Vgl. u.a. Rundschreiben 1946, S. 4f.; NDV 33 (1953), S. 255. In NDV 28 (1948), S. 196f., wurde darauf verwiesen, daß die meisten Fürsorgeempfänger gegenwärtig entweder zur alten Gruppe der gehobenen Fürsorge oder zu neuen Betroffenengruppen gehörten, also alle nach den Grundsätzen der gehobenen Fürsorge betreut werden müßten und eine fürsorgerische Gruppenbildung von daher ohnehin obsolet sei.

203 Vgl. dazu generell Hockerts, Vorsorge.

204 Rundschreiben 1946, S.51. Eine detailliertere Rekonstruktion alliierter Vorhaben und deutscher Reaktionen liegt bisher leider nicht vor. Vgl. den Überblick bei Willing, Vorgeschichte, S.610f.; ferner Heisig, Armenpolitik, 1995, S.30ff., dem Willing, Vorgeschichte, S.609, Anm. 113, allerdings Recherchefehler nachweist. Heisig hat zwar einschlägige Aktenbestände des Bundesarchivs Koblenz benutzt, benennt seine Quellen aber nur summarisch, verschweigt bedauerlicherweise u.a. den Inhalt des Kontrollratsplans und bewegt sich in einem schematisierenden Interpretationsrahmen, wonach ein deutscher „Fürsorgekomplex“ der „Bedrohung“ alliierter Reformpläne „von oben“ durch Reformen „von unten“ begegnen wollte, so daß seine Darstellung insgesamt wenig befriedigt. 
die Wiederherstellung bzw. Sicherung der Rechtseinheit ein Gebot der Stunde war, meinten auch deutsche Fürsorgepolitiker, war dies doch unabdingbare Voraussetzung für einen funktionierenden Ausgleich der Kriegsfolgelasten. Darüber hinaus herrschte auch bei der Fürsorge erhebliche Rechtsunsicherheit, hervorgerufen durch das Nebeneinander alter und neuer Rechtssetzungsinstanzen, die unterschiedlichen Vorgaben der Besatzungsmächte, durch Zonen- und neue Ländergrenzen ${ }^{205}$ und auch durch die Unklarheit darüber, welche Bestandteile des alten, während der NS-Zeit durch zahlreiche Sonderbegünstigungen ergänzten Rechts noch weiter galten, und welche nicht. Ein rechtlich und regional kleinteiliges soziales Sicherungssystem wie die öffentliche Fürsorge mußte in einer Zeit starker Wanderungsbewegungen eine mögliche Rechtszersplitterung umso empfindlicher treffen.

Einheitliche Trägerschaft und klare örtliche Zuständigkeit, wurde argumentiert, bildeten die Grundlage nicht nur für die Sicherung der finanziellen Leistungsfähigkeit der Fürsorgeverbände, sondern auch dafür, daß der Flüchtling, Kriegsheimkehrer oder umherwandernde Jugendliche überall vor Ort die notwendige Hilfe auch tatsächlich erhielt. Diese Hilfe müßte überall in Zielsetzung, Form und Umfang die gleiche, das materielle Fürsorgerecht also prinzipiell einheitlich sein, da ein untragbares Leistungsgefälle sozial ungerecht sei und „wohl auch eine neue ständige Wanderungsbewegung unter der Bevölkerung und damit auch eine Minderung ihrer Arbeitswilligkeit auslösen könnte“206. Eine solche Argumentation mußte auch zunächst widerstrebende Kommunalpolitiker auf die Seite der Verteidiger eines überregional einheitlichen Fürsorgerechts ziehen. Wie allerdings diese Einheitlichkeit zu erreichen sei, darüber herrschten zwischen Militärregierungen und deutschen Fürsorgefachleuten zunächst offensichtlich unterschiedliche Ansichten, wenn auch alle Seiten an möglichst geringen Fürsorgekosten interessiert waren. ${ }^{207}$

Angesichts der späteren Entwicklung in der SBZ zielten die sowjetischen Pläne im Kontrollrat vermutlich auf einen Abbau individualisierender, vorbeugender und nachgehender Hilfen sowie auf eine zentrale Steuerung der Fürsorge zu Lasten der Städte und Landkreise. ${ }^{208}$ Das mußte, zumal nach den Erfahrungen unter dem Nationalsozialismus, auf westdeutscher Seite die Verfechter der kommunalen Selbstverwaltungsrechte und des fürsorgerischen Individualprinzips auf den Plan rufen, die allemal im neu formierten DV zusammentrafen. Nachdem im Kontroll-

205 Im neuen Land Hessen etwa galt für einen Teil des Landes das preußische Ausführungsgesetz zur RFV, in dem anderen das Landesrecht des ehemaligen Freistaates Hessen; vgl. Rundschreiben 1946, S. $49 f$.

206 Sorg, Aufgaben, S. 50. Vgl. Rundschreiben 1946, S. 37; Polligkeit u.a. in der ersten Nachkriegsausgabe des NDV, Rundschreiben 1946, S. 2; Entschließung des Deutschen Fürsorgetags vom 16.6.1947, in: NDV 27 (1947), S. 62.

$207 \mathrm{Vgl}$. Willing, Vorgeschichte, S. $617 f$.

208 Nach Einführung einer eigenen Sozialfürsorge in der SBZ im Frühling 1947 sei, so der DV-Referent Krug von Nidda, „russischerseits“ vorgeschlagen worden, die Regelung der SBZ auf die drei übrigen Besatzungszonen und Berlin auszudehnen; vgl. Krug von Nidda, „Vorschläge zu einer Fürsorgerechtsreform“ vom 30.9.1947. Ms., LAB, B Rep. 142-9, 1282. Vgl. auch Lehmann, Sozialversicherung, S. V. 
rat offensichtlich die Fürsorgereformpläne aufgrund des Desinteresses der westlichen Besatzungsmächte nach einem Jahr noch keine nennenswerten Fortschritte gemacht hatten, bat im Oktober 1946 schließlich der Vertreter der amerikanischen Militärregierung im Wohlfahrtsausschuß des Zonenbeirats um Reformvorschläge von deutscher Seite. ${ }^{209}$ Ein daraufhin im Auftrag des hessischen Wohlfahrtsministeriums erstelltes Gutachten Polligkeits befürwortete nur kleinere Änderungen zur Anpassung an aktuelle Bedürfnisse.210 Dies war möglich, da das Weimarer Fürsorgerecht die Zeit des Nationalsozialismus formal weitgehend unverändert überstanden hatte und Amerikaner und Briten nicht nur das RJWG in seiner ursprünglichen Fassung für anwendbar erklärten, sondern sogar das Gesetz über den öffentlichen Gesundheitsdienst von 1934 bestehen ließen. Eine Gesamtreform der RFV und der RGr., so Polligkeits Gutachten, sei zur Zeit aufgrund der ungeklärten wirtschaftlichen, finanziellen und staatsrechtlichen Lage Deutschlands weder möglich noch nötig. Das wichtigste Problem, die Sicherung der finanziellen Leistungsfähigkeit der Fürsorgeträger, könne für eine Übergangszeit auch jeweils in den Ländern geregelt werden. Für die im Interesse der Rechtseinheit „unbedingt notwendigen Änderungen des Fürsorgerechts“ genüge eine „Teilreform“: Die Einheitsfürsorge sollte allgemein verbindlich werden und ausschließlich auf Grundlage der RFV und deren Ausführungsbestimmungen durchgeführt werden. ${ }^{211}$ Die Richtsätze sollten im Rahmen der kommunalen Selbstverwaltung von den Bezirksfürsorgeverbänden festgesetzt werden und sich für zusammenhängende Wirtschaftsgebiete an Richtlinien der obersten Landesbehörden orientieren; damit wandte sich Polligkeit gegen die Bestrebungen zahlreicher Länder, die Kontrolle der Richtsätze wieder stärker an sich zu ziehen. Die Neufassung der RGr. und ihre Eingliederung in die RFV müsse einer späteren Gesamtreform vorbehalten werden; die Bestimmungen zur "gehobenen Fürsorge" seien aber schon jetzt außer Kraft zu setzen. Unbedingt aufrechtzuerhalten seien hingegen die Prinzipien der Individualisierung und der Nachrangigkeit der Fürsorgeleistungen. Außerdem müßten neben der Methode der Familienfürsorge „unter den Wirkungen der Kriegsfolgen als volkswichtig zu bezeichnende Aufgaben “212 ergänzend festgeschrieben werden: verstärkte Arbeitsfürsorge für Körperbehinderte, Erziehung und Erwerbsbefähigung Minderjähriger und Mitwirkung bei der aufbauenden Gesundheitsfürsorge. Durchgesetzt werden sollte die Reform aber nicht über ein Kontrollratsgesetz, sondern durch Vereinbarungen der Länder über gleichgerichtete Gesetze. Polligkeits Begründung, für ein Kontrollratsgesetz sei es zu früh, erscheint etwas bemüht angesichts der virulenten Schwierigkeiten, die Ländergesetzgebung zu koordinieren. ${ }^{213}$ Noch am 7. Mai 1946 hatte der Länderrat einem

${ }^{209}$ Vgl. Willing, Vorgeschichte, S. 610.

210 Gutachten auszugsweise veröffentlicht in: Rundschreiben 1946, S. 49ff.; vgl. auch Willing, Vorgeschichte, S. 606.

211 Hier versuchte Polligkeit, das alte DV-Ziel formalrechtlich festzuzurren, indem er u.a. behauptete, die Aufzählung einzelner Hilfsbedürftigengruppen in $\mathbb{1}$ RFV sei nur aus formalen Gründen erklärlich, und deren weitergehende Implikationen verschwieg; vgl. Rundschreiben 1946, S. 50.

212 Ebenda, S. 51.

213 So selbst ein ungezeichneter Artikel ebenda, S. 37; ferner Sorg, Aufgaben, S. 52. 
Antrag des Sozialpolitischen Ausschusses, in den drei Ländern der US-Zone das Fürsorgewesen einheitlich zu regeln, nur in stark abgeschwächter Form zugestimmt. ${ }^{214}$ Polligkeit ging es jedoch vermutlich vielmehr darum, den Einfluß der deutschen Seite (und nicht zuletzt den eigenen) zu maximieren und einen möglicherweise von den sowjetischen Vorstellungen stark beeinflußten alliierten Oktroi zu verhindern. ${ }^{215}$ Der DV-Vorsitzende bediente sich der gleichen Argumentation wie die Gegner der alliierten Reformpläne für die Sozialversicherung, was wenig überrascht, da Polligkeit selbst an einflußreicher Stelle zu deren Gruppe zählte. ${ }^{216}$

Eine gemeinsame Reform durch den Kontrollrat schien ohnehin aus deutscher Sicht seit dem Frühling 1947 obsolet, denn drei Monate nach ihrem Alleingang bei der Sozialversicherung beschritt die sowjetische Militärverwaltung in enger Zusammenarbeit mit der Deutschen Verwaltung für Arbeit und Sozialfürsorge (DVAS) auch in der komplementären öffentlichen Fürsorge eigene Wege ${ }^{217}$ : Im Mai 1947 wurden RFV und RGr. formell aufgehoben und durch unmittelbar verbindliches Zonenrecht über „Sozialfürsorge“ ersetzt, dessen Modalitäten eine Verordnung vom September des gleichen Jahres genauer regelte. ${ }^{218}$ Ziel, so die offizielle Begründung der DVAS, sei es, „statt des Gestrüpps der bisherigen unzähligen Bestimmungen [...] eine klare zeitgemäße Regelung zu schaffen“219. Das neue Recht knüpfte mit dem Grundsatz der Nachrangigkeit der Fürsorge sowie materiellrechtlich noch eng an das bisherige Recht an, beschnitt aber bereits deutlich die Kompetenzen der Länder und vor allem der Kommunen und begründete damit eine stärkere systemkonforme Zentralisierung und Standardisierung der Fürsorge. ${ }^{220}$

214 Vgl. Tagung des Länderrats am 7.5.1946, in: Akten zur Vorgeschichte, Bd.1, S. 491f.

215 Polligkeit erklärte auch öffentlich vor dem Deutschen Fürsorgetag 1947, bei einem Kontrollratsgesetz sei „es oft nicht leicht [...], die deutschen Belange [...] ausreichend zur Geltung zu bringen“, Kriegsfolgenhilfe, S.21.

216 Polligkeit war einer von fünf Sachverständigen, die im Oktober 1946 in einem Gutachten für die drei Ministerpräsidenten der US-Zone eine umfassende Reform der Sozialversicherung für verfrüht erklärten und für eine weitgehende Beibehaltung des bisherigen Systems plädierten; dazu wie zu den von anderen Gegnern der Sozialversicherungsreform angeführten Gründen vgl. Hockerts, Entscheidungen, S. 55ff.

217 Vermutlich war das ebensowenig wie im Falle der Sozialversicherung eine Mißachtung der bisherigen Kontrollratsverhandlungen, sondern der Versuch, durch ein fait accompli die gemeinsame Verabschiedung der Beratungsergebnisse zu forcieren; vgl. ebenda, S. 67f.; Hoffmann, Neuordnung, S. 69.

218 Vgl. Befehl Nr. 92 des Obersten Chefs der SMAD vom 22.4.1947, mit dem die „Verordnung über Sozialfürsorge für die Bevölkerung in der sowjetischen Besatzungszone Deutschlands“ der DVAS zum 15.5.1947 in Kraft gesetzt wurde, ZVOBl. 1947, Nr. 5, S.54; ferner die „1.Durchführungsverordnung zur Verordnung über Sozialfürsorge vom 22.4.1947 gemäß Befehl Nr.92 des Obersten Chefs der SMAD“ der DVAS vom 2.9.1947, ZVOBl. 1947, Nr.19, S.219. Vgl. auch allgemein Hoffmann, Neuordnung, S. $161 \mathrm{ff}$.

219 Zitiert in: NDV 27 (1947), S. 72.

220 Vgl. ebenda, S. $71 \mathrm{ff}$.; 28 (1948), S. 20ff.; Boldorf, Sozialfürsorge in der SBZ/DDR und ders., Sozialfürsorge in der SBZ; zur weiteren Entwicklung Rudloff, Fürsorge, S. $201 \mathrm{ff}$. Träger der Sozialfürsorge blieben die Stadt- und Landkreise bzw. die Länder, wobei die fürsorgerechtliche Lastenzuständigkeit erheblich vereinfacht wurde. Eine wesentliche Neuerung betraf die Richtsätze: Richtlinien der DVAS legten im September 1947 neue, 
Einerseits stärkte das neue Recht die Stellung des Hilfsbedürftigen, indem prinzipiell ein Rechtsanspruch auf Fürsorge eingeräumt, die Rückzahlungspflicht abgeschafft und die Heranziehung Unterhaltspflichtiger abgemildert wurde; andererseits wurde der Zwang zum Einsatz der eigenen Arbeitskraft erheblich verschärft. Darüber hinaus spiegelte schon diese frühe Variante der Sozialfürsorge die neuen ideologischen Gegebenheiten, wenn ehemalige „Führer und aktive[n] Mitglieder der NSDAP und ihrer Gliederungen, ehemalige[n] Militaristen, Junker, Inhaber von Konzernen, Trusts und anderen Arten von Monopolen, sowie Personen, deren Vermögen nach dem 8. Mai 1945 infolge verbrecherischer faschistischer Tätigkeit beschlagnahmt worden ist", von der Sozialfürsorge ausgeschlossen wurden. ${ }^{221}$ Fürsorgerechtliches Pendant dieser Regelung war eine neue "gehobene“ Fürsorge, die für (anerkannte!) „Opfer des Faschismus“ höhere Richtsätze und bevorzugte Behandlung vorsah.

Hatte der Kommentator im Nachrichtendienst des DV im Februar 1948 noch gemeint, für die neuen Regelungen hätten RFV und RGr. nur modifiziert, nicht aber aufgehoben werden müssen, so wurde spätestens im September des gleichen Jahres deutlich, daß das neue Zonenrecht auf eine rigidere Handhabung und zentralisierte Regelung der Fürsorge im Dienste der Arbeitskräftebeschaffung zielte: Eine Anordnung der Deutschen Wirtschaftskommission verschärfte u.a. die Voraussetzungen für Hilfsbedürftigkeit, beschränkte die vorbeugende Hilfe auf Maßnahmen zur Erwerbsbefähigung und definierte eine Höchstgrenze für tatsächlich gezahlte Unterstützungen. ${ }^{222}$ Für kommunale Willkür bei der Unterstützungsbemessung blieb in der SBZ damit kaum noch Raum, für kommunale Innovation und vertiefte individuelle Hilfeleistung allerdings auch nicht. Die Entwicklung der öffentlichen Fürsorge in der SBZ und frühen DDR entsprach damit der zentralistischen Umgestaltung des gesamten politischen Systems, die 1952/53 schließlich die letzten Residuen kommunaler Selbstverwaltung ausschaltete. ${ }^{223}$

Während somit in der sowjetischen Besatzungszone seit dem Frühling 1947 die Rechtseinheit auf der Basis eines vereinfachten, auf dem Verwaltungswege erlassenen Fürsorgerechts wiederhergestellt war, plädierten westdeutsche Fürsorgevertreter weiterhin fast gebetsmühlenartig dafür, den Status quo wenigstens in den drei westlichen Besatzungszonen beizubehalten und nötige Modifikationen in (west-)deutscher Eigenregie über Länderkoordination durchzuführen, wie es dann allerdings nur im Falle der Fürsorgerechtsvereinbarung auch wirklich funk-

höhere und zoneneinheitliche Richtsätze fest, definierten deren Höchstgrenze und Zusammensetzung und erreichten so eine weitgehende Standardisierung, wenn auch auf niedrigem Niveau; vgl. „Richtlinien für die Bemessung der Barleistungen für den Lebensunterhalt eines Hilfsbedürftigen" vom 2. 9.1947, ZVOBl. 1947, Nr. 19.

221 Verordnung über Sozialfürsorge vom 2.9.1947, Ziffer 2.

222 „Anordnung zur Durchführung der Verordnung über Sozialfürsorge und des SMADBefehls Nr. 92/1947“ der Deutschen Wirtschaftskommission für die sowjetische Besatzungszone vom 21.9.1948 mit „Richtlinien für die Bemessung der Barleistung für den Lebensunterhalt eines Hilfsbedürftigen“ vom 8.10.1948, ZVOBl. 1948 Nr.45, S.469, 500. Vgl. ferner NDV 29 (1949), S. $66 f$.

223 Vgl. Ritter, Über Deutschland, S. 139. 
tionierte. ${ }^{224}$ Es ist anzunehmen, daß ähnlich wie bei der Sozialversicherung ${ }^{225}$ die amerikanische und die britische Militärregierung schließlich die Regelung dieser komplizierten sozialpolitischen Materie gerne künftigen deutschen Stellen überließen und im Zuge des sich verschärfenden Ost-West-Konflikts eher gewillt waren, auf die vorherrschende westdeutsche Meinung Rücksicht zu nehmen. Vor allem Polligkeits Festhalten an den Weimarer Fürsorgegesetzen entsprang aber mit Sicherheit nicht nur einer Abwehrhaltung gegen alliierte Reformabsichten; vielmehr ging es ihm auch um eine Wahrung des seinerzeit erreichten einheitlichen Niveaus der Fürsorge und der Leistungsfähigkeit ihrer Träger, die es nicht zuletzt gegenüber kommunalen Selbständigkeitsbestrebungen oder finanziell überforderten Flüchtlingsländern zu bewahren galt. Nur auf der Basis eines einheitlichen Fürsorgerechts, mahnte der DV-Vorsitzende, sei die „Überwindung der deutschen Volksnot" möglich, für die das gesamte deutsche Volk die Verantwortung übernehmen müsse 226; das hieß im Klartext: Wer einen wirksamen Lastenausgleich der Kriegsfolgenhilfe wolle, müsse ein Interesse an einem einheitlichen Fürsorgerecht tragen und auf lokale Eigenmächtigkeiten oder Länder- und Gruppenegoismen verzichten.

Noch aber waren die institutionellen Voraussetzungen für eine auch nur bizonale einheitliche Änderung des Fürsorgerechts kaum gegeben, verfügte der neue Wirtschaftsrat in Frankfurt doch über keinerlei diesbezügliche Kompetenzen. Neun Tage vor der Konstituierung der Bizonen-Legislative forderte daher der erste reguläre Nachkriegs-Fürsorgetag am 16. Juni 1947 für den Wirtschaftsrat auch die Gesetzgebungsbefugnis für die Sozial- und Fürsorgepolitik. ${ }^{227}$ Die von den Fürsorgespezialisten im DV und den kommunalen Spitzenverbänden in den folgenden Monaten immer wieder erhobene Forderung nach einer zentralen Regelungsinstanz ${ }^{228}$ entsprach durchaus der Logik der deutschen Fürsorgegeschichte, in der die Weimarer Verfassung dem Reich für Armenwesen und Wohlfahrtspflege Gesetzgebungskompetenzen zugestanden und damit eine reichsweit einheitlich konzipierte Unterstützungspflicht und die Schaffung leistungsfähiger Fürsorgeverbände ermöglicht hatte. Doch während die Amerikaner und Briten im Laufe des Jahres 1948 auf dem Gebiet der Sozialversicherung und des Arbeitsrechts deutschem Drängen nachgaben, dem Parlamentarischen Rat entsprechende Kompetenzen übertrugen und eine eigene bizonale Verwaltung für Arbeit errichteten $^{229}$, blieb die Regelung des Fürsorgewesens ausschließlich Ländersache und eine Koordination damit außerordentlich schwierig.

Parallel zu den Bemühungen um die Wahrung der Rechtseinheit arbeiteten Länderministerien einerseits, die DV-Führung und die kommunalen Spitzenverbände

224 Vgl. entsprechende Entschließungen des DST-Vorstands vom 17.5.1947 und des Deutschen Fürsorgetages vom 16.6.1947, NDV 27 (1947), S.62, der neuen Arbeitsgemeinschaft der LFV der Bizone von 29./30.8.1947, ebenda, S.82, und des Vorstands des Hessischen Gemeindetags vom 1.9.1947, Die Selbstverwaltung 1 (1947), S. 45.

225 Vgl. Hockerts, Entscheidungen, S. $79 \mathrm{ff}$.

226 Polligkeit, Fürsorgerecht, S. $26 f$.

227 Vgl. NDV 27 (1947), S. 62.

228 Vgl. ebenda, S. 101; 28 (1948), S. 62, $68 f$.

229 Vgl. Hockerts, Entscheidungen, S. $107 \mathrm{ff}$. 
andererseits an einer zumindest regionalen Angleichung der Richtsätze, denn nach Kriegsende hatten die Kommunen deren Festsetzung gerne wieder in eigene Regie übernommen, zahlten in vergleichbaren Fällen zum Teil sehr unterschiedliche Unterstützungen und unterliefen so das mit dem Richtsatzerlaß von 1941 erreichte national vereinheitlichte Mindestniveau. ${ }^{230}$ Mit der weitgehenden Übernahme der Kriegsfolgenhilfelasten, die ja auf den Fürsorgerichtsätzen basierten, verstärkte sich aber anders als Mitte der zwanziger Jahre nun auch das Interesse der Länder an deren einheitlicher Gestaltung, und die meisten Sozialministerien erließen seit 1946/47 entsprechende Richtlinien. Eine solche Rückkehr zu zentraler Regulierung widersprach zwar kommunalpolitischer Tradition ${ }^{231}$; nichtsdestoweniger beteiligten sich die kommunalen Spitzenverbände wiederholt an der Ausarbeitung solcher Länderrichtlinien, zumal die Richtsätze schon im Interesse der Länder selbst eher knapp bemessen waren und die Vorgaben den kommunalen Trägern in der Regel „einen ausreichenden Spielraum“232 ließen, so daß ihr Zustandekommen von der DV-Führung ,im Interesse der Hilfsbedürftigen und vom Standpunkt des Finanzausgleichs" 233 begrüßt wurde. Mit solchen regionalen Richtlinien hofften führende DV- und Kommunalvertreter, die Richtsätze im Interesse „sparsame[r] Bewirtschaftung der Fürsorgemittel“ möglichst „einer unsachlichen Beeinflussung politischer Stellen “234 auf lokaler Ebene entziehen zu können und zu verhindern, „daß die Kreise ihre Richtsätze gegenseitig in die Höhe treiben“235. Gleichzeitig konnten auf diese Weise „schwarze Schafe“ unter den Kreisen und Städten zu verbesserten Leistungen verpflichtet und damit parlamentarischer Kritik der Wind aus den Segeln genommen werden. Und nicht zuletzt waren solche einheitlichen Richtsatzvorgaben dazu geeignet, Wünschen nach gruppenfürsorgerischer Sonderbehandlung auch in der Praxis leichter zu begegnen.

Auf den erwähnten hessischen Richtsatz-Erlaß vom Februar 1946 folgte ein Erlaß des nordrhein-westfälischen Sozialministers vom Mai 1947, der höhere Mindest- und Höchstrichtsätze vorschrieb, und Niedersachsen und SchleswigHolstein erließen bald ebenfalls entsprechende Regelungen.236 In Bayern hatte das Innenministerium im April 1947 in einer allgemeinen Anweisung Mindestrichtsätze empfohlen, die Festsetzung höherer Sätze aber weiterhin den Bezirksfürsorgeverbänden überlassen.237 Im Sommer 1947 forderte der Sozialpolitische Aus-

230 Vgl. Heisig, Armenpolitik, 1995, S. 33ff.

231 Zur Opposition der kommunalen Spitzenverbände, unterstützt vom DV, gegen den zunächst nur formalen Richtsatzzwang 1926 vgl. Leibfried, Existenzminimum, passim; Sachße/Tennstedt, Geschichte, Bd.2, S.179ff.; zum Widerstand gegen den Richtsatzerlaß von 1941 vgl. Leibfried u.a., Geteilte Erde?, passim.

232 NDV 27 (1947), S. 121.

233 Ebenda, S. 125.

234 Ebenda, S. 122.

235 So der Landkreisverband Bayern, NDV 29 (1949), S.250. Tatsächlich waren es nicht selten die unmittelbar betroffenen Kreisdirektoren oder Landräte selbst, die eine Erhöhung der Richtsätze forderten, da sonst nicht einmal die auf den Lebensmittelkarten vorgesehenen Rationen erworben werden könnten. Beispiele aus Nordrhein-Westfalen zitiert bei Heisig, Armenpolitik, 1995, S.37f.

236 Vgl. zu den Details auch NDV 27 (1947), S. 121ff.; 29 (1949), S. $6 f$.

237 Vgl. NDV 27 (1947), S. 73. 
schuß des Zonenbeirats der britischen Zone sogar länder- und zonenübergreifende Richtsätze, da nur so ein einheitliches Vorgehen gegenüber den Besatzungsmächten und ein angemessener Lastenausgleich möglich seien. ${ }^{238}$ Von diesem Ziel war man jedoch auch Ende 1948 noch deutlich entfernt: So betrug der Richtsatz für einen Familienvater in Hamburg $40 \mathrm{DM}$, während für seinen Nachbarn in Elmshorn $29 \mathrm{DM}$ zugrunde gelegt wurden.239 Anders als in der sowjetischen Zone blieb den Ländern und Fürsorgeverbänden der drei Westzonen damit nach 1945 ein relativ großer Spielraum für die Bemessung des zum Lebensbedarf Notwendigen, der erst mit den Verwaltungsvorschriften über den Aufbau der Richtsätze vom Dezember 1955 eingeschränkt wurde.

Rechtliche Sonderentwicklungen gab es schließlich in einigen fürsorgerischen Spezialbereichen: Zur Eindämmung der sich sprunghaft ausbreitenden Geschlechtskrankheiten erließen verschiedene Länder zunächst besondere Verordnungen und Anweisungen, die sich aber nicht wesentlich von dem noch gültigen „Reichsgesetz zur Bekämpfung der Geschlechtskrankheiten“ von 1927 entfernten ${ }^{240}$; nachdem die vier Besatzungsmächte im Mai 1947 zum Schutz der eigenen Armeeangehörigen sämtliche Länder zur Verabschiedung entsprechender neuer Gesetze aufgefordert hatten ${ }^{241}$, folgten die SBZ sowie Hamburg, Bremen und Niedersachsen 1948/49 mit verstärkten Zwangsmaßnahmen. ${ }^{242} \mathrm{Zu}$ einer (bundes-) einheitlichen Regelung dieses Komplexes kam es erst mit dem fürsorgerische und sozialpädagogische Gesichtspunkte stärker betonenden „Bundesgesetz zur Bekämpfung der Geschlechtskrankheiten" vom 23. Juli 1953, als das Problem bereits weit weniger virulent war. Die Bekämpfung der anderen großen Nachkriegsseuche, der Tuberkulose, wurde in einzelnen Ländern modifiziert, ohne daß die ebenfalls noch gültige „Verordnung über Tuberkulosehilfe“ vom 8. September 1942 grundsätzlich verändert worden wäre. 243

Als heikel erwiesen sich schließlich die Versuche Hessens und vor allem Bayerns, die zwangsweise "Unterbringung“ und „Arbeitserziehung“ von nichtseßhaften, geschlechtskranken oder anderweitig "gefährdeten“ Jugendlichen und jungen Erwachsenen in besonderen Anstalten zu ermöglichen. ${ }^{244}$ Nachdem unter den Sachverständigen des Länderrats der amerikanischen Zone keine Einigung über eine „Verordnung zum Schutze der heimatlosen Jugend“ erzielt werden konnte, hatte dessen Rechtsausschuß den Erlaß jeweils eigener Länderverordnun-

238 Vgl. Heisig, Armenpolitik, 1995, S. 38.

239 Vgl. NDV 29 (1949), S. 6.

240 Vgl. für Hessen: „Verordnung zur Bekämpfung der Geschlechtskrankheiten“ vom 11.4. 1946, GVBl. für Groß-Hessen vom 10.5.1946, S.110; für Schleswig-Holstein und Württemberg-Hohenzollern: NDV 27 (1947), S. 137.

241 Vgl. Direktive Nr. 52, in: Amtsblatt des Kontrollrats in Deutschland Nr. 15, S. 91.

242 Vgl. NDV 28 (1948), S. 34; 29 (1949), S. 133ff.; 30 (1950), S. 20f.; Brackmann/Drilling, Wohlfahrtspflege, S. $124 f$.

243 Vgl. Rundschreiben 1946, S. 41f.; NDV 27 (1947), S. 134ff.; 28 (1948), S. $32 \mathrm{ff}$.

244 „Verordnung zum Schutz der heimatlosen Jugend“ vom 23.3.1946, GVBl. für GroßHessen vom 11.6.1946, S.135; „Verordnung Nr.73 zum Schutz der heimatlosen Jugendlichen“, „Verordnung Nr. 74 zur Unterbringung verwahrloster Frauen und Mädchen“, „Verordnung Nr.75 über Arbeitserziehung“, sämtlich vom 15.4.1946, Bayerisches GVBl. 20.8.1946, S. 218 f. 
gen empfohlen. ${ }^{245}$ Während die hessische Verordnung neben Familienzusammenführung, Unterbringung in Lehr- und Pflegestellen ein Festhalterecht der Jugendämter in geeigneten Anstalten und Fürsorgeerziehung bis zum 21. Lebensjahr vorsah, begründeten die ähnlichen bayerischen Verordnungen neben einem Abschiebeverbot eine Festhaltepflicht und sahen für junge geschlechtskranke oder „sonst sittlich verwahrloste“ Frauen sowie für nicht-arbeitswillige Jugendliche bis 25 Jahre die Möglichkeit einer Zwangseinweisung in „Arbeitserziehungsheime“ vor. ${ }^{246}$ Mit diesen Verordnungen wie auch mit den teils geforderten, teils zunächst verwirklichten verschärften Zwangsmaßnahmen gegen Geschlechtskranke oder "Arbeitsscheue“ war erneut das schon in der Weimarer Republik heftig diskutierte Thema einer fürsorgerisch begründeten Freiheitsentziehung bis hin zur „Bewahrung“ angeschlagen, das dann im Mittelpunkt der Diskussionen um ein „Bewahrungsgesetz“ in den fünfziger Jahren stehen sollte.

Angesichts der weiterhin latenten Zersplitterung des Fürsorgerechts plädierten DV-Vorstand und kommunale Spitzenverbände bei den Vorarbeiten für das Grundgesetz dafür, die öffentliche Fürsorge ähnlich wie in den Jahren der Weimarer Republik möglichst uneingeschränkt in die „Vorranggesetzgebung“ des Bundes einzubeziehen ${ }^{247}$ und schlossen sich den Protesten gegen die Pläne der Militärgouverneure an, die Vorrangkompetenzen des Bundes generell stark zu beschneiden, da dies für die öffentliche Fürsorge ein Rückschritt vor die Zeit des Unterstützungswohnsitzgesetzes von 1870 sei. ${ }^{248}$ Auch die Sozialminister der Länder wollten (wie der Herrenchiemseer Entwurf) zumindest die Regelung der Grundsätze der öffentlichen Fürsorge dem Bund vorbehalten, da der Fürsorgelastenausgleich einigermaßen vergleichbare Leistungen voraussetze. ${ }^{249}$ Anders als im Falle der Sozial- und der Arbeitslosenversicherung war die legislative Kompetenzverteilung für die öffentliche Fürsorge im Parlamentarischen Rat durchaus umstritten: Hier stand das vor allem von seiten der CSU verfolgte Ziel, den Ländern und Kommunen möglichst weitgehende Gestaltungsmöglichkeiten zu bewahren und den Bund allenfalls auf Rahmenvorschriften zu beschränken, im Konflikt mit dem Interesse an einer einheitlichen, effizienten Fürsorgepolitik, das für die Vertreter der SPD wie der CDU im Vordergrund stand. ${ }^{250}$ Schließlich konnte sich letztere Auffassung durchsetzen und schuf durch die ausdrückliche und uneingeschränkte Einbeziehung der „öffentlichen Fürsorge“ in den Gegenstandskatalog der „konkurrierenden Gesetzgebung“

245 Vgl. Bamberger, Kampf, S. 56f. In der britischen Zone hatten die Landesjugendämter Ende 1945 in den „Nenndorfer Richtlinien“ die Beschaffung und Finanzierung der notwendigen Einrichtungen für heimatlose Jugendliche übernommen ; vgl. Hasenclever, Jugendhilfe, S. 156.

246 Vgl. auch Rundschreiben 1946, S. 9f. Die bayerischen Verordnungen über Arbeitserziehung wurden Ende 1947 wieder aufgehoben, da der durch sie begründete Eingriff in die persönliche Freiheit verfassungswidrig war; vgl. NDV 27 (1947), S. 132.

247 So NDV 28 (1948), S. $195 f$.

248 Vgl. Schreiben des Vorstandes des DV an den Parlamentarischen Rat vom 25.3.1949, in: NDV 29 (1949), S. $61 \mathrm{f}$.

$249 \mathrm{Vgl}$. ebenda, S. 86.

250 Vgl. Münch, Sozialpolitik, S. 82ff. 
(Art. 74 GG Ziff. 7) wieder die Voraussetzungen für die künftige Verabschiedung eines einheitlichen Bundesfürsorgegesetzes. „Auf dieser Basis“, konstatierte befriedigt Anfang November der Fürsorgespezialist des Evangelischen Hilfswerks, Paul Collmer, habe „die föderalistische Linie in der Fürsorgegesetzgebung nicht die Aussicht, Blüten zu treiben“.251 Denn bis auf weiteres galt das alte Reichsrecht in Form von RFV, RGr. und RJWG als Bundesrecht weiter (Art. 125 GG) und konnte nur durch die Bundesorgane, nicht aber mehr einzelne Länder geändert werden. ${ }^{252}$

Bei welchem Bundesminister die öffentliche Fürsorge ressortieren sollte, war keineswegs von Anfang an klar, denn sowohl für eine Ansiedelung in einem Bundesinnenministerium wie in einem Bundesarbeitsministerium konnten gleichermaßen historische Gründe geltend gemacht werden. ${ }^{253}$ Zudem bestand ein enger Zusammenhang mit der Frage, welche Bundesminister für die Flüchtlinge, das Gesundheitswesen und den sozialen Wohnungsbau zuständig sein würden. Der die künftige Ressortverteilung planende Organisationsausschuß der Ministerpräsidentenkonferenz sprach sich am 10. Juli 1949 mit überwiegender Mehrheit dafür aus, die Fürsorge einem umfassenden Arbeits- und Sozialministerium zu unterstellen. ${ }^{254}$ Eine Minderheit im Ausschuß befürwortete - wie die kommunalen Spitzenverbände und der DV - die Unterbringung im Bundesinnenministerium, das engere Verbindungen zu den Kommunen als den Trägern der Fürsorge besitze. ${ }^{255}$

251 [Paul Collmer:] „Vortrag für die Tagung über Fragen des Fürsorgerechts in Bremen-Lesum am 1. und 2. November 1949“, Ms., ADW, Allg. Slg., B. 931 I.

252 Vgl. NDV 29 (1949), S. 155f. Zur zentralen Bedeutung des Artikels 125 GG, der gerade in der sozialpolitischen Gesetzgebung die traditionelle Dominanz des Reiches fortführte, vgl. Münch, Sozialpolitik, S. 91, 101f.

253 Aus armenpolizeilicher Tradition heraus waren oberhalb der Gemeinden die staatlichen Innenverwaltungen für die Armenfürsorge zuständig gewesen; 1922 übertrug der Reichstag die Zuständigkeit für die Wohlfahrtspflege einschließlich der Armenfürsorge (ohne Jugendfürsorge und -pflege) vom Reichsministerium des Innern auf das Reichsarbeitsministerium, das nun ein alle Hilfsbedürftigen einbeziehendes, nicht diskriminierendes System der Wohlfahrtspflege initiieren sollte; weder das Reichsinnenministerium noch offensichtlich der kommunal orientierte DV sahen diese Kompetenzverlagerung gern; vgl. Sachße/Tennstedt, Geschichte, Bd.2, S.145f.; Hong, Welfare, S.116f. Nach 1933 konnte das Innenministerium wesentliche Kompetenzen zurückgewinnen: Seit 1934 für den neuen staatlichen öffentlichen Gesundheitsdienst zuständig, wurden ihm 1935 auch die allgemeine Fürsorge, die Wandererfürsorge und die freie Wohlfahrtspflege wieder unterstellt; vgl. Sachße/Tennstedt, Geschichte, Bd. 3, S. 29.

254 Vgl. Protokoll der 13. Sitzung des Organisationsausschusses am 10.7.1949, in: Akten zur Vorgeschichte, Bd. 5, S. 828. Der künftige Arbeitsminister Storch hingegen plädierte für ein eigenes Wohlfahrtsministerium, da er ein „klassisches“ Arbeitsministerium (zuständig für Arbeitsrecht, Arbeitsschutz, Arbeitsvermittlung, Sozialversicherung und Kriegsopferversorgung) favorisierte; vgl. Protokoll der 9. Sitzung am 2.7.1949, in: ebenda, S. 736; ferner Hockerts, Entscheidungen, S.110f.

255 Vgl. v.a. die Äußerungen des bayerischen Ministerialdirektors Ringelmann, in: Akten zur Vorgeschichte, Bd.5, S. 825, 827f. (teilweise irrtümliche Vertauschung von Arbeitsund Innenministerium durch den Protokollanten). Die gemeinsamen Vorschläge der Arbeitsgemeinschaft der Kommunalen Spitzenverbände und des DV „Zur Organisation der Bundesregierung“ in: KommBl 1 (1949), H. 18, S.1f. 
Tatsächlich ressortierte die öffentliche Fürsorge dann im zunächst von Gustav Heinemann (CDU) geleiteten Bundesministerium des Innern. ${ }^{256}$ Ausschlaggebend dafür dürfte in erster Linie gewesen sein, daß Adenauer sich schließlich gegen die Bildung eines „großen“ Arbeits- und Sozialministeriums entschied ${ }^{257}$ und damit die öffentliche Fürsorge mehr oder weniger automatisch wieder dem Innenressort zufiel.258 Diese Entscheidung war für die Entwicklung der öffentlichen Fürsorge mehr als nur eine formal-organisatorische: Sie verfestigte die sachliche Trennung der Fürsorge von den anderen Systemen der sozialen Sicherung und förderte mit Sicherheit ihr Dasein als "Stiefkind“ der Sozialpolitik wie auch des jeweiligen Ressortleiters. Gleichzeitig begünstigte diese Entscheidung kommunale Interessen; wenn auch das Innenministerium nicht mehr mit der Kommunalaufsicht betraut war, verstand es sich doch als Sachwalter kommunaler Belange bei der Gesetzgebung gegenüber Ländern und anderen Bundesressorts. ${ }^{259}$ Nicht zuletzt sicherte die Zuordnung dem DV und seinem Vorsitzenden Gehör und Einfluß, der vor allem auf alten Kontakten und Zusammenarbeit im ehemaligen Reichsinnenministerium und im Deutschen Gemeindetag beruhte. ${ }^{260}$

$256 \mathrm{Zu}$ Aufbau und Zuständigkeit des BMI vgl. die offiziöse Darstellung von Faude/Fritz, Bundesministerium, S. 22ff. Zu den Anfangsschwierigkeiten vgl. Kitz, Aufgaben, S. 244f.

$257 \mathrm{Zu}$ den Gründen vgl. Hockerts, Entscheidungen, S. 110f.

258 Die Sozialabteilung des BMI wurde auch für die „Kriegsfolgenhilfe“ federführend.

259 Vgl. Keßler, Bundesministerium. Der ehemalige Landrat und 1944 in die Kommunalabteilung des Reichsinnenministeriums berufene Erich Keßler war nach dem Krieg zunächst für die Abwicklungsstelle des Rechnungshofs in der britischen Zone tätig und gehörte seit Ende 1948 einem kleinen, von Adenauer initiierten CDU-nahen Gremium an, das Personalvorschläge für die künftigen Bundesministerien erarbeiten sollte. Auf diese Weise gewann Keßler, der sich auch sehr für die Berufung Hans Globkes eingesetzt hatte, entscheidenden Einfluß auf die Erstbesetzung des BMI, das im Gegensatz zu den übrigen großen Bundesministerien nicht auf einer bizonalen Vorläuferinstitution aufbauen konnte; vgl. Morsey, Personal- und Beamtenpolitik, S. 203, 208f., 221f.; Oppelland, Gerhard Schröder, S. 261. Keßler wurde im neuen BMI Leiter der Unterabteilung für Verwaltung, Verwaltungsgerichtsbarkeit und Kommunalwesen; vgl. Heisig, Armenpolitik, 1990, S. 549f. Vgl. auch die beinahe euphorische Darstellung der engen Zusammenarbeit zwischen kommunalen Spitzenverbänden und leitenden Beamten des BMI bei Groeben/Heide, Geschichte, S. 262ff.

260 Der erste Leiter der Sozialabteilung im BMI, Wilhelm Kitz (1890-1956), hatte bis 1945 als Erster Landesrat in der Rheinischen Landesverwaltung u.a. gute Kontakte zum DGT-Beigeordneten Georg Schlüter. Nach dem Krieg an leitender Stelle im nordrheinwestfälischen Finanzministerium tätig, war er bis zu seinem Eintritt in das BMI im Februar 1950 u.a. Generalsekretär der Gutachterkommission für den Lastenausgleich in Bad Homburg, Vorsitzender des Trizonalen Ausschusses für Gemeindefinanzen sowie Mitglied im von Polligkeit geleiteten Arbeitsstab „Kriegsfolgenhilfe“. Dem DV-Hauptausschuß gehörte er seit 1949 an; vgl. NDV 36 (1956), S. 279ff.; Heisig, Armenpolitik, 1990, S. 550f. Kitz' Nachfolger Gerhard Scheffler (1894-1977) war bis 1939 in der Kommunalabteilung des Reichsinnenministeriums tätig gewesen. Dort hatten auch der spätere DV-Vorsitzende Hans Muthesius sowie der spätere DV-Referent Krug von Nidda bis Kriegsende gearbeitet, vgl. Heisig, Armenpolitik, 1990, S. 558, 567ff., 584f. Georg Schlüter wiederum, der während des Krieges eng mit Muthesius zusammengearbeitet hatte, wurde 1947 stellvertretender Hauptgeschäftsführer des DLT und war seit 1946 Mitglied des DV, dessen Wiedergründung er gefördert hatte; vgl. ebenda, S. 587; Groeben/Heide, Geschichte, S.231, 294. 
Das waren ganz andere Konstellationen als in den zwanziger Jahren, als das stark von der katholischen Soziallehre geprägte Reichsarbeitsministerium mit seinen engen Kontakten zu den freien Wohlfahrtsverbänden für die Fürsorge zuständig gewesen war.

Ende der vierziger Jahre war für die öffentliche Fürsorge die Phase des unmittelbaren Wiederaufbaus beendet: Nach vorübergehender „Allzuständigkeit“ hatte sie einen Teil ihrer Zuständigkeiten an die nach und nach in den Ländern wieder errichtete Renten- und Arbeitslosenversicherung abgetreten oder würde sie nun in die neuen Versorgungssysteme überführen. Dies wie der allmählich einsetzende wirtschaftliche Aufschwung mußten bald zu einem Bedeutungsverlust der öffentlichen Fürsorge beitragen, der sich bereits jetzt quantitativ in einem Sinken der Zahl der Empfänger wie der Ausgaben widerspiegelte.

Mit der Verabschiedung des Grundgesetzes und der Zuordnung der öffentlichen Fürsorge zum Innenressort waren nach der kommunalen und länderweisen nun auch die zentrale Zuständigkeit und Gesetzgebungskompetenz auf Bundesebene wieder klar geregelt und gleichzeitig die bundesweite Gültigkeit des aus der Weimarer Republik tradierten Fürsorgerechts gesichert. Gleichzeitig war ab 1950 auch die umfassende Regelung der Kriegsfolgenhilfe als dem zentralen Problem einer finanziellen Konsolidierung der Fürsorgeträger in die Wege geleitet, nachdem die Ausdehnung der FRV seit Juli 1949 die fürsorgerechtliche Lastenverteilung geklärt hatte. Schließlich war 1949 auch die trizonale bzw. bundesweite Verbandsbildung sowohl auf seiten der Kommunen wie auf seiten der freien Wohlfahrtspflege in enger Anlehnung an Weimarer Vorbilder abgeschlossen. Die eigentliche Nachkriegszeit war damit, einmal überspitzt formuliert, für das System der öffentlichen Fürsorge vorbei. 